Document downloaded from:

http://hdl.handle.net/10251/121419

This paper must be cited as:

Cortés, J.; Navarro-Quiles, A.; Romero, J.; Roselló, M. (2018). Computing the probability density function of non-autonomous first-order linear homogeneous differential equations with uncertainty. Journal of Computational and Applied Mathematics. 337:190-208. https://doi.org/10.1016/j.cam.2018.01.015

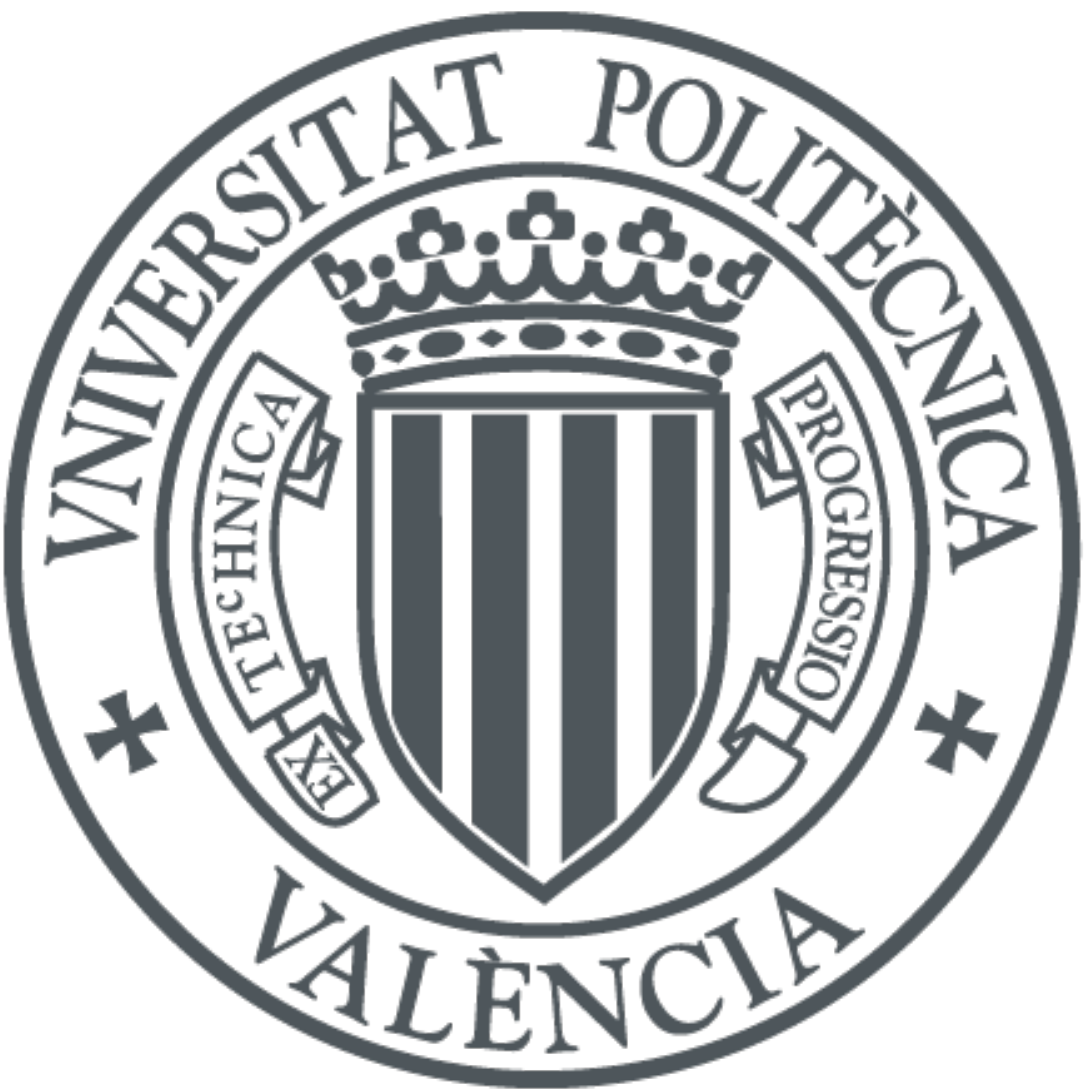

The final publication is available at

http://doi.org/10.1016/j.cam.2018.01.015

Copyright Elsevier

Additional Information 


\title{
Computing the probability density function of non-autonomous first-order linear homogeneous differential equations with uncertainty
}

\author{
J.-C. Cortés ${ }^{\mathrm{a}}$, A. Navarro-Quiles ${ }^{\mathrm{a}}$, J.-V. Romero ${ }^{\mathrm{a}}$, M.-D. Roselló ${ }^{\mathrm{a}, *}$ \\ ${ }^{a}$ Instituto Universitario de Matemática Multidisciplinar, \\ Universitat Politècnica de València, \\ Camino de Vera s/n, 46022, Valencia, Spain
}

\begin{abstract}
This paper is devoted to construct approximations of the probability density function of the nonautonomous first-order homogeneous linear random differential equation, where the initial condition and the diffusion coefficient are assumed to be a random variable and a stochastic process, respectively. We combine Random Variable Transformation technique and Karhunen-Loève expansion to construct reliable approximations under general conditions. Several numerical examples illustrate our theoretical findings.
\end{abstract}

Keywords: Karhunen-Loève expansion, Random Variable Transformation technique, first probability density function, random first-order non-autonomous linear differential equation

\section{Introduction and motivation}

It is well-known that the derivative of a function plays a key role to measure instantaneous changes of a quantity of interest. This fact justifies the ubiquity of differential equations in the realm of Mathematical Modelling. There exist numerous problems in Physics, Chemistry, Epidemiology, Engineering, Economics, etc., that are formulated by differential equations. When these equations are put forward in practice, their input data are usually fixed using information that is often contaminated of uncertainty because two main reasons. On the one hand, uncertainty can be attributed due to the inherent complexity that is often involved in many physical phenomena. On the other hand, errors and uncertainties are introduced by numerical algorithms and experimental data used to approximate and calibrate mathematical models. As a consequence, these models are often formulated by randomizing classical (deterministic) differential equations. In this regard, it is important to stress that this randomization can mainly be made using two different approaches [1, Sec. 4.7]. First, Stochastic Differential Equations (SDEs) where uncertainty is forced by a stochastic process having irregular sample behaviour. This is the case of Itô-type SDEs, where randomness is considered by the so-called White Noise

\footnotetext{
*Corresponding author

Email addresses: jccortes@imm.upv.es (J.-C. Cortés), annaqui@doctor.upv.es (A. Navarro-Quiles), jvromero@imm.upv.es (J.-V. Romero), drosello@imm.upv.es (M.-D. Roselló) 
stochastic process. This process results from the derivative (in a generalized sense based upon the theory of distributions) of a Gaussian stochastic process termed Wiener process (or Brownian Motion) whose trajectories are continuous but nonwhere differentiable. The Wiener process is a particular case of diffusion processes, thereby Markovian, whose increments are stationary, independent and Gaussian. The cornerstone tool for handling this class of SDEs is the so-called Itô lemma, which can be interpreted as a chain rule for differentiating diffusion stochastic processes. The solutions of these SDEs exhibit nondifferentiable sample paths because the irregularity of the driving Wiener process. Exhaustive studies related to both ordinary and partial SDEs from different perspectives, theoretical, computational, numerical and applications, can be found in $[2,3,4,5]$, respectively. Secondly, Random Differential Equations (RDEs) are those in which random effects are directly manifested in input parameters (coefficients, source/forcing term and initial/boundary conditions). These inputs are often assumed to possess milder behaviour like continuity with respect to time and/or space. However, at this point it is interesting to stress that even somewhat irregular random functions, as the Wiener process, are still allowed to play the role of inputs data under this approach. This issue will be illustrated through some examples later. RDEs have another important advantage for modelling purposes, since apart from Gaussian patterns, further probabilistic distributions can be assumed for random inputs including Binomial, Poisson, Beta, Gamma, Lognormal, etc., for instance. In this context, the powerful classical differential equations are randomized to better describe physical problems (in a wide sense). This approach is implemented by assuming that constants and/or functions playing the role of input data are random variables (RVs) and stochastic processes (SPs), respectively. References $[1,6,7,8,9]$ provide an excellent overview about the foundations on RDEs and their main analytical/numerical techniques

It is important to point out that the rigorous analysis of SDEs and RDEs usually takes place in the Hilbert space $\mathrm{L}^{2}\left(\Omega, \mathrm{L}^{2}(\mathcal{T}, \mathrm{H})\right)$ of square integrable SPs, that are valued on a Hilbert space $\left(\mathrm{H},\langle\cdot, \cdot\rangle_{\mathrm{H}}\right)$, and these SPs are defined over an underlying complete probability space $(\Omega, \mathcal{F}, \mathbb{P})$. Let us introduce the mathematical ingredients that will be required to develop rigorously our working context:

- A complete probability space $(\Omega, \mathcal{F}, \mathbb{P})$ whose elements $\omega$ are termed events.

- The Hilbert space $\left(\mathrm{H},\langle\cdot, \cdot\rangle_{\mathrm{H}}\right)$. For reasons that will be apparent later, elements of $\left(\mathrm{H},\langle\cdot, \cdot\rangle_{\mathrm{H}}\right)$ will be denoted by $x(t, \omega)$. Then,

$$
\mathrm{H}=\left\{x(t, \omega):\|x(t, \omega)\|_{\mathrm{H}}=+\sqrt{\langle x(t, \omega), x(t, \omega)\rangle_{\mathrm{H}}}<+\infty,(t, \omega) \in \mathcal{T} \times \Omega\right\} .
$$

- The Hilbert space $\left(\mathrm{L}^{2}(\mathcal{T}, \mathrm{H}),\langle\cdot, \cdot\rangle_{\mathrm{L}^{2}(\mathcal{T}, \mathrm{H})}\right), \mathcal{T} \subset \mathbb{R}$, defined as

$$
\mathrm{L}^{2}(\mathcal{T}, \mathrm{H})=\left\{x(\cdot, \omega): \mathcal{T} \longrightarrow \mathrm{H}:\|x(\cdot, \omega)\|_{\mathrm{L}^{2}(\mathcal{T}, \mathrm{H})}<+\infty\right\},
$$

where

$$
\langle x(\cdot, \omega), y(\cdot, \omega)\rangle_{\mathrm{L}^{2}(\mathcal{T}, \mathrm{H})}=\int_{\mathcal{T}}\langle x(t, \omega), y(t, \omega)\rangle_{\mathrm{H}} \mathrm{d} t,
$$

and

$$
\begin{aligned}
\|x(\cdot, \omega)\|_{\mathrm{L}^{2}(\mathcal{T}, \mathrm{H})} & =+\sqrt{\langle x(\cdot, \omega), x(\cdot, \omega)\rangle_{\mathrm{L}^{2}(\mathcal{T}, \mathrm{H})}}=\left(\int_{\mathcal{T}}\langle x(t, \omega), x(t, \omega)\rangle_{\mathrm{H}} \mathrm{d} t\right)^{1 / 2} \\
& =\left(\int_{\mathcal{T}}\left(\|x(t, \omega)\|_{\mathrm{H}}\right)^{2} \mathrm{~d} t\right)^{1 / 2}<+\infty . \\
2 & <+\infty
\end{aligned}
$$


50 From these ingredients, one defines the Hilbert space

$$
\mathrm{L}^{2}\left(\Omega, \mathrm{L}^{2}(\mathcal{T}, \mathrm{H})\right)=\left\{x: \mathcal{T} \times \Omega \longrightarrow \mathrm{L}^{2}(\mathcal{T}, \mathrm{H}):\|x\|_{\mathrm{L}^{2}\left(\Omega, \mathrm{L}^{2}(\mathcal{T}, \mathrm{H})\right)}<+\infty\right\},
$$

51

52

with the following inner product

$$
\begin{aligned}
\langle x, y\rangle_{\mathrm{L}^{2}\left(\Omega, \mathrm{L}^{2}(\mathcal{T}, \mathrm{H})\right)} & =\int_{\Omega}\langle x(t, \omega), y(t, \omega)\rangle_{\mathrm{L}^{2}(\mathcal{T}, \mathrm{H})} \mathrm{d} \mathbb{P}(\omega)=\int_{\Omega} \int_{\mathcal{T}}\langle x(t, \omega), y(t, \omega)\rangle_{\mathrm{H}} \mathrm{d} t \mathrm{~d} \mathbb{P}(\omega) \\
& =\int_{\mathcal{T}} \int_{\Omega}\langle x(t, \omega), y(t, \omega)\rangle_{\mathrm{H}} \mathrm{d} \mathbb{P}(\omega) \mathrm{d} t=\int_{\mathcal{T}} \mathbb{E}\left[\langle x(t, \omega), y(t, \omega)\rangle_{\mathrm{H}}\right] \mathrm{d} t,
\end{aligned}
$$

and norm

$$
\|x\|_{\mathrm{L}^{2}\left(\Omega, \mathrm{L}^{2}(\mathcal{T}, \mathrm{H})\right)}=\left(\int_{\mathcal{T}} \mathbb{E}\left[\left(\|x(t, \omega)\|_{\mathrm{H}}\right)^{2}\right] \mathrm{d} t\right)^{1 / 2}<+\infty,
$$

where the Fubbini's theorem has been applied to express both, the inner product and the norm, in terms of expectation operator. So far, we have carefully distinguished in notation the elements $x$ and $x(t, \omega)$, but, for the sake of convenience, henceforth we will write them indistinctly.

As throughout this paper we will consider real RVs and SPs, we will take $\mathrm{H}=\mathbb{R}$ endowed with the standard inner product $\langle x, y\rangle_{\mathbb{R}}=x y$, with $x, y \in \mathbb{R}$. In this case $\mathrm{L}^{2}\left(\Omega, \mathrm{L}^{2}(\mathcal{T}, \mathbb{R})\right)$ is usually denoted as $\mathrm{L}^{2}\left(\Omega, \mathrm{L}^{2}(\mathcal{T})\right)$. Moreover, we will assume that $\mathcal{F}=\mathcal{B}(\mathbb{R})$ is the Borel $\sigma$ algebra on $\mathbb{R}$. Therefore, we will work in the Hilbert space $\mathrm{L}^{2}\left(\Omega, \mathrm{L}^{2}(\mathcal{T})\right)$ with the inner product $\langle x, y\rangle_{\mathrm{L}^{2}\left(\Omega, \mathrm{L}^{2}(\mathcal{T})\right)}=\int_{\mathcal{T}} \mathbb{E}[x(t, \omega) y(t, \omega)] \mathrm{d} t$ and whose elements are SPs such that $\int_{\mathcal{T}} \mathbb{E}\left[(x(t, \omega))^{2}\right] \mathrm{d} t<$ $+\infty$. In practice, an important case, that will be considered throughout our subsequent development, is when $\mathcal{T}$ is a bounded and closed interval of the real line, $\mathcal{T}=\left[t_{0}, T\right] \subset \mathbb{R}$. In that case, second-order RVs, that is RVs $x(\omega)$ with finite variance (and hence $\mathbb{E}\left[(x(\omega))^{2}\right]<+\infty$ ), are obviously elements of the Hilbert space $\mathrm{L}^{2}\left(\Omega, \mathrm{L}^{2}(\mathcal{T})\right)$. These RVs are interpreted as constant SPs. In dealing with sequences of second-order RVs, the above inner product defines a norm whose associate convergence is usually referred to as mean square convergence. Apart from this convergence, the study of SDEs and RDEs can be developed considering another types of stochastic convergences such as almost surely convergence, convergence in probability and convergence in distribution, and using the relationship among them as well.

Throughout this paper we will deal with non-autonomous first-order linear RDEs. As a major difference with respect to its deterministic counterpart, solving a RDE means not only to compute its exact/approximate solution SP but also calculating its main statistical functions such as the expectation and the variance. It is important to highlight that even in the case of linear RDEs, the achievement of these goals does not just consist of generalizing its classical counterpart. To support this assertion down below we exhibit some illustrative examples in this respect that are aimed to motivate our interest in studying non-autonomous first-order linear RDEs. Indeed, important results, well-known in the deterministic framework, are satisfied in the random scenario only under restrictive assumptions. For instance, if we consider the autonomous first-order linear RDE with deterministic initial condition

$$
x^{\prime}(t, \omega)=a(\omega) x(t, \omega), \quad t \geq 0 ; \quad x(0, \omega)=1, \quad \omega \in \Omega,
$$

where $a \equiv a(\omega)$ is a second-order RV, it can be shown that the extension to the random scenario of the classical existence and uniqueness Picard's theorem is satisfied if, and only if, $a$ is bounded almost surely [6, p.119], [10]. As a consequence, this important result is not applicable when $a$ assumes a Gaussian or a Poisson distribution, for instance. As a second illustrative example 
involving the computation of the expectation of the solution SP to non-autonomous linear RDEs, let us consider the random initial value problem (IVP)

$$
x^{\prime}(t, \omega)=a(t, \omega) x(t, \omega)+b(t, \omega), \quad x\left(t_{0}, \omega\right)=x_{0}(\omega), \quad \omega \in \Omega,
$$

where $a(t, \omega)$ and $b(t, \omega)$ are second-order SPs (i.e., $\mathbb{E}\left[(a(t, \omega))^{2}\right]$ and $\mathbb{E}\left[(b(t, \omega))^{2}\right]$ are finite for all $t$ ) and $a_{0}(\omega)$ is a second-order RV. If $\mathbb{E}[\cdot]$ denotes the expectation operator, then it can be shown that the mean of the solution, $\mu_{x}(t)=\mathbb{E}[x(t, \omega)]$, does not satisfy the corresponding averaged ordinary differential equation

$$
\frac{\mathrm{d} \mu_{x}(t)}{\mathrm{d} t} \neq \mathbb{E}[a(t, \omega)] \mu_{x}(t)+\mathbb{E}[b(t, \omega)], \quad \mu_{x}\left(t_{0}\right)=\mathbb{E}\left[x_{0}(\omega)\right] .
$$

Instead the computation of the expectation $\mu_{x}(t)$ is more involved (see, [6, Ch.8] and [11, p.66]). The earlier examples illustrate the challenges when dealing with both theoretical and practical aspects regarding linear RDEs. In this latter regard, it is important to emphasize that besides calculating the first statistical moments, the computation of the first probability density function (1-PDF), say $f_{1}(x, t)$, of the solution SP is much more desirable since, from it, one can compute all one-dimensional statistical moments including, as particular cases, both the mean and the variance

$$
\mu_{x}(t)=\mathbb{E}[x(t, \omega)]=\int_{-\infty}^{\infty} x f_{1}(x, t) \mathrm{d} x, \quad \sigma_{x}^{2}(t)=\mathbb{V}[x(t, \omega)]=\int_{-\infty}^{\infty} x^{2} f_{1}(x, t) \mathrm{d} x-\left(\mu_{x}(t)\right)^{2} .
$$

Furthermore, the 1-PDF provides a comprehensive probabilistic description of the solution SP for each fixed time instant $t$ and it permits to calculate the probability that the solution SP lies on a specific set of interest as well

$$
\mathbb{P}[a \leq x(t, \omega) \leq b]=\int_{a}^{b} f_{1}(x, t) \mathrm{d} x .
$$

The computation of the 1-PDF of the solution SP for the linear RDE has been recently undertaken by some of the authors in [12]. In this contribution one develops a comprehensive probabilistic study to the general linear RDE in the case that input data (diffusion coefficient, forcing term and initial condition) are assumed to be RVs, i.e., the analysis is just carried out for the autonomous linear RDE. This study is based on the application of the so-called Random Variable Transformation (RVT) technique in order to obtain the 1-PDF of the solution SP. RVT technique is stated in Th. 1. In the context of ordinary and partial RDEs and their applications, this technique has been applied with the same objective [13, 14, 15, 16, 17].

Theorem 1 (Random Variable Transformation technique). Let $\mathbf{x}(\omega)=\left[x_{1}(\omega), \ldots, x_{m}(\omega)\right]^{\top}$ and $\mathbf{y}(\omega)=\left[y_{1}(\omega), \ldots, y_{m}(\omega)\right]^{\top}$ be two m-dimensional absolutely continuous random vectors defined on a complete probability space $(\Omega, \mathfrak{F}, \mathbb{P})$. Let $\mathbf{r}: \mathbb{R}^{m} \rightarrow \mathbb{R}^{m}$ be a one-to-one deterministic transformation of $\mathbf{x}(\omega)$ into $\mathbf{y}(\omega)$, i.e., $\mathbf{y}(\omega)=\mathbf{r}(\mathbf{x}(\omega)), \omega \in \Omega$. Assume that $\mathbf{r}$ is a continuous mapping and has continuous partial derivatives with respect to each component $x_{i}, 1 \leq i \leq m$. Then, if $f_{\mathbf{x}}\left(x_{1}, \ldots, x_{m}\right)$ denotes the joint probability density function of vector $\mathbf{x}(\omega)$, and $\mathbf{s}=\mathbf{r}^{-1}=\left(s_{1}\left(y_{1}, \ldots, y_{m}\right), \ldots, s_{m}\left(y_{1}, \ldots, y_{m}\right)\right)$ represents the inverse mapping of $\mathbf{r}=\left(r_{1}\left(x_{1}, \ldots, x_{m}\right), \ldots, r_{m}\left(x_{1}, \ldots, x_{m}\right)\right)$, the joint probability density function of vector $\mathbf{y}(\omega)$ is given by

$$
f_{\mathbf{y}}\left(y_{1}, \ldots, y_{m}\right)=f_{\mathbf{x}}\left(s_{1}\left(y_{1}, \ldots, y_{m}\right), \ldots, s_{m}\left(y_{1}, \ldots, y_{m}\right)\right)\left|\mathcal{J}_{m}\right|,
$$


where $\left|\mathcal{J}_{m}\right|$, which is assumed to be different from zero, denotes the absolute value of the jacobian defined by the determinant

$$
\mathcal{J}_{m}=\operatorname{det}\left[\begin{array}{ccc}
\frac{\partial s_{1}\left(y_{1}, \ldots, y_{m}\right)}{\partial y_{1}} & \ldots & \frac{\partial s_{m}\left(y_{1}, \ldots, y_{m}\right)}{\partial y_{1}} \\
\vdots & \ddots & \vdots \\
\frac{\partial s_{1}\left(y_{1}, \ldots, y_{m}\right)}{\partial y_{m}} & \ldots & \frac{\partial s_{m}\left(y_{1}, \ldots, y_{m}\right)}{\partial y_{m}}
\end{array}\right] .
$$

In this paper, we go further by attacking the non-autonomous case for the homogeneous linear RDE whose initial condition is assumed to be a RV. Specifically, hereinafter we shall consider the following random IVP

$$
\left.\begin{array}{l}
x^{\prime}(t, \omega)=a(t, \omega) x(t, \omega), \quad t \in \mathcal{T}=\left[t_{0}, T\right] \subset \mathbb{R}, \\
x\left(t_{0}, \omega\right)=x_{0}(\omega)
\end{array}\right\}
$$

where

$$
\text { H1 : } x_{0}(\omega) \text { is a second-order RV and } a(t, \omega) \in \mathrm{L}^{2}\left(\Omega, \mathrm{L}^{2}(\mathcal{T})\right),
$$

satisfying certain additional conditions that will be specified later. As we assume that $x_{0}(\omega)$ is a second-order $\operatorname{RV}\left(\mathbb{E}\left[\left(x_{0}(\omega)\right)^{2}\right]=k_{0}<+\infty\right)$ and $\mathcal{T}=\left[t_{0}, T\right]$ has finite volume, then $x_{0}(\omega) \in$ $\mathrm{L}^{2}\left(\Omega, \mathrm{L}^{2}(\mathcal{T})\right)\left(\left\|x_{0}\right\|_{\mathrm{L}^{2}\left(\Omega, \mathrm{L}^{2}(\mathcal{T})\right)}=\left(\int_{\mathcal{T}} \mathbb{E}\left[\left(\left\|x_{0}(\omega)\right\|_{\mathrm{H}}\right)^{2}\right] \mathrm{d} t\right)^{1 / 2}=\sqrt{k_{0}\left(T-t_{0}\right)}<+\infty\right)$.

The main goal of this paper is to obtain the 1-PDF, $f_{1}(x, t)$, of the solution SP, $x(t, \omega)$, to the random IVP (2). To achieve this objective, we will take advantage of combining the application of the RVT technique (see Th.1) and the Karhunen-Loève expansion (KLE), that is stated in Th. 2. KLE is a type-Fourier series method that allows us to represent the diffusion SP in (2), $a(t, \omega)$, as a function of a denumerable set of second-order RVs $\left\{\xi_{i}(\omega): i \geq 1\right\}$ such that they have zero mean $\left(\mathbb{E}\left[\xi_{i}(\omega)\right]=0\right)$, unit variance $\left(\mathbb{V}\left[\xi_{i}(\omega)\right]=1\right)$ and are pairwise uncorrelated $\left(\mathbb{E}\left[\xi_{i}(\omega) \xi_{j}(\omega)\right]=0\right.$ if $\left.i \neq j\right)$. In other words, $\left\{\xi_{i}(\omega): i \geq 1\right\}$ are such that $\mathbb{E}\left[\xi_{i}(\omega)\right]=0$ and $\mathbb{E}\left[\xi_{i}(\omega) \xi_{j}(\omega)\right]=\delta_{i j}$, where $\delta_{i j}$ denotes the standard Kronecker delta function.

Theorem 2 ( $\mathrm{L}^{2}$ convergence of Karhunen-Loève). [3, p.202] Consider a mean square integrable continuous time stochastic process $x \equiv\{x(t, \omega): t \in \mathcal{T}, \omega \in \Omega\}$, i.e., $x \in \mathrm{L}^{2}\left(\Omega, \mathrm{L}^{2}(\mathcal{T})\right)$ being $\mu_{x}(t)$ and $c_{x}(s, t)$ its mean and covariance functions, respectively. Then,

$$
x(t, \omega)=\mu_{x}(t)+\sum_{j=1}^{\infty} \sqrt{v_{j}} \phi_{j}(t) \xi_{j}(\omega), \quad \omega \in \Omega,
$$

converges in $\mathrm{L}^{2}\left(\Omega, \mathrm{L}^{2}(\mathcal{T})\right)$, where

$$
\xi_{j}(\omega):=\frac{1}{\sqrt{v_{j}}}\left\langle x(t, \omega)-\mu_{x}(t), \phi_{j}(t)\right\rangle_{\mathrm{L}^{2}(\mathcal{T})},
$$

and $\left\{\left(v_{j}, \phi_{j}(t)\right): j \geq 1\right\}$ denote, respectively, the eigenvalues with $v_{1} \geq v_{2} \geq \cdots \geq 0$ and eigenfunctions of the following integral operator $\mathfrak{C}$

$$
(\mathfrak{C} f)(t):=\int_{\mathcal{T}} c_{x}(s, t) f(s) \mathrm{d} s, \quad f \in \mathrm{L}^{2}(\mathcal{T}),
$$

associated to the covariance function $c_{x}(s, t)$. Random variables $\xi_{j}(\omega)$ have zero mean, unit variance and are pairwise uncorrelated. Furthermore, if $x(t, \omega)$ is Gaussian, then $\xi_{j}(\omega) \sim \mathrm{N}(0,1)$ are independent and identically distributed. 
To keep the computational burden feasible, later when we apply the RVT technique to compute approximations of the 1-PDF, $f_{1}(x, t)$, we will need consider the $N$-truncation of infinite $\operatorname{sum}(3)$

$$
x_{N}(t, \omega)=\mu_{x}(t)+\sum_{j=1}^{N} \sqrt{v_{j}} \phi_{j}(t) \xi_{j}(\omega), \quad \omega \in \Omega .
$$

Therefore, the $N+1$ second-order $\operatorname{RVs} x_{0}(\omega)$ and $\left\{\xi_{i}(\omega): 1 \leq i \leq N\right\}$ will be involved. In this manner, we will obtain the 1-PDF, $f_{1}^{N}(x, t)$, of $x_{N}(t, \omega)$ rather than the exact 1-PDF, $f_{1}(x, t)$, of $x(t, \omega)$. In our subsequent analysis, we will provide conditions in order to guarantee the convergence of $f_{1}^{N}(x, t)$ to $f_{1}(x, t)$ as $N \rightarrow+\infty$.

The paper is organized as follows. The aim of Section 2 is twofold, first to compute the 1-PDF, $f_{1}^{N}(x, t)$, of the truncated solution SP, $x_{N}(t, \omega)$, given in (4) and, secondly, to provide sufficient conditions in order to guarantee the convergence of the 1-PDF, $f_{1}^{N}(x, t)$, to the exact solution SP, $x(t, \omega)$, as $N \rightarrow+\infty$. In Section 3, two numerical examples will be shown to illustrate the theoretical results established in Section 2. Our conclusions are drawn in Section 4.

\section{Computing the 1-PDF of the truncated solution stochastic process}

It is known that the exact closed solution SP to the random IVP (2) is

$$
x(t, \omega)=x_{0}(\omega) \operatorname{Exp}\left[\int_{t_{0}}^{t} a(s, \omega) \mathrm{d} s\right], \quad \omega \in \Omega .
$$

It is important to note that given a SP, say $a(t, \omega)$, in general nothing is known about the probabilistic distribution of the following SP, $\hat{a}(t, \omega)=\int_{0}^{t} a(s, \omega) \mathrm{d} s$. An exception is when $a(t, \omega)$ is a Gaussian SP. In that case, it can be proved that $\hat{a}(t, \omega)$ is also Gaussian, see [6, Th. 4.64, p.112]. As we are interested in the determination of the 1-PDF of the SP (5) in the general case that the SP $a(t, \omega)$ has an arbitrary probabilistic distribution (hence no Gaussian in general), we will take advantage of combining KLE and RVT techniques to give an answer to this interesting question under mild conditions.

Our analysis will be carried out assuming that the initial condition $x_{0}(\omega)$ is a RV such as, for every $t \in \mathcal{T}=\left[t_{0}, T\right]$ fixed, $x_{0}(\omega)$ and $a(t, \omega)$ are independent RVs. Observe that this assumption is realistic from a practical standpoint when dealing with physical models since initial conditions and coefficients involved in the differential equations are not often physically related. Anyway, at this point we stress that our subsequent analysis can also be carry out if independence between $x_{0}(\omega)$ and $a(t, \omega)$ is not embraced. As a consequence, if we denote by $\xi_{N}(\omega)=\left(\xi_{1}(\omega), \ldots, \xi_{N}(\omega)\right)$ the random vector whose components are the RVs arising in the KLE of $a(t, \omega)$, then we will assume that $x_{0}(\omega)$ and $\xi_{N}(\omega)$, are independent. Additionally, we will suppose that $x_{0}(\omega)$ is an absolutely continuous RV and $\xi_{N}(\omega)$ is an absolutely continuous random vector whose PDFs will be denoted by $f_{0}\left(x_{0}\right)$ and $f_{\xi_{N}}\left(\xi_{1}, \ldots, \xi_{N}\right)$, respectively. If we denote by $\boldsymbol{\xi}_{N+1}=\left(x_{0}(\omega), \xi_{1}(\omega), \ldots, \xi_{N}(\omega)\right)$, observe that due to independence between $x_{0}(\omega)$ and $\boldsymbol{\xi}_{N}(\omega)$, their joint PDF, $f_{\xi_{N+1}}\left(x_{0}, \xi_{1}, \ldots, \xi_{N}\right)$, is the product of their marginal PDFs, i.e.,

$$
f_{\xi_{N+1}}\left(x_{0}, \xi_{1}, \ldots, \xi_{N}\right)=f_{0}\left(x_{0}\right) f_{\xi_{N}}\left(\xi_{1}, \ldots, \xi_{N}\right) .
$$


Summarizing, in the following we will assume that

$x_{0}(\omega), \xi_{i}(\omega), 1 \leq i \leq N$, are absolutely continuous RVs.

H2 : $\quad x_{0}(\omega), \boldsymbol{\xi}_{N}(\omega)=\left(\xi_{1}(\omega), \ldots, \xi_{N}(\omega)\right)$ are independent

with PDFs $f_{0}\left(x_{0}\right)$ and $f_{\xi_{N}}\left(\xi_{1}, \ldots, \xi_{N}\right)$, respectively.

Moreover, $\mathbb{E}\left[\xi_{i}(\omega)\right]=0$ and $\mathbb{E}\left[\xi_{i}(\omega) \xi_{j}(\omega)\right]=\delta_{i j}$.

177 Let us assume that $a \equiv a(t, \omega)$ is a continuous time SP such that $a \in \mathrm{L}^{2}\left(\Omega, \mathrm{L}^{2}(\mathcal{T})\right)$ and let $\mu_{a}(t)$

178 and $c_{a}(s, t)$ denote its mean and covariance functions, respectively. According to Th. 2 , the SP $a(t, \omega)$ admits a KLE, and let us consider its truncation of order $N$ (see expression (4))

$$
a_{N}(t, \omega)=\mu_{a}(t)+\sum_{j=1}^{N} \sqrt{v_{j}} \phi_{j}(t) \xi_{j}(\omega), \quad \omega \in \Omega
$$

Therefore substituting this expression in (5), a formal approximate solution SP to the random IVP (2) is given by

$$
x_{N}(t, \omega)=x_{0}(\omega) \operatorname{Exp}\left[\int_{t_{0}}^{t} a_{N}(s, \omega) \mathrm{d} s\right]=x_{0}(\omega) \operatorname{Exp}\left[\int_{t_{0}}^{t}\left(\mu_{a}(s)+\sum_{j=1}^{N} \sqrt{v_{j}} \phi_{j}(s) \xi_{j}(\omega)\right) \mathrm{d} s\right] .
$$

Now, we will apply the RVT technique, stated in Th. 1, to obtain the 1-PDF of the approximate solution SP (7) in terms of the PDFs $f_{0}\left(x_{0}\right)$ and $f_{\xi_{N}}\left(\xi_{1}, \ldots, \xi_{N}\right)$, which are assumed known. As the RVT method applies to RVs, we first fix $t \in \mathcal{T}=\left[t_{0}, T\right]$ and then we consider the following mapping $\mathbf{r}: \mathbb{R}^{N+1} \rightarrow \mathbb{R}^{N+1}$

$$
\begin{array}{ccc}
y_{1}= & r_{1}\left(x_{0}, \xi_{1}, \ldots, \xi_{N}\right)=x_{0} \operatorname{Exp}\left[\int_{t_{0}}^{t}\left(\mu_{a}(s)+\sum_{j=1}^{N} \sqrt{v_{j}} \phi_{j}(s) \xi_{j}\right) \mathrm{d} s\right], \\
y_{2}= & r_{2}\left(x_{0}, \xi_{1}, \ldots, \xi_{N}\right)=\xi_{1}, \\
\vdots & \\
y_{N+1}= & r_{N+1}\left(x_{0}, \xi_{1}, \ldots, \xi_{N}\right)=\xi_{N},
\end{array}
$$

86 whose inverse transformation $\mathbf{s}=\mathbf{r}^{-1}$ is

$$
\begin{gathered}
x_{0}=s_{1}\left(y_{1}, y_{2}, \ldots, y_{N+1}\right)=y_{1} \operatorname{Exp}\left[-\int_{t_{0}}^{t}\left(\mu_{a}(s)+\sum_{j=1}^{N} \sqrt{v_{j}} \phi_{j}(s) y_{j+1}\right) \mathrm{d} s\right], \\
\xi_{1}=s_{2}\left(y_{1}, y_{2}, \ldots, y_{N+1}\right)=y_{2}, \\
\vdots \\
\xi_{N}=s_{N+1}\left(y_{1}, y_{2}, \ldots, y_{N+1}\right)=y_{N+1} .
\end{gathered}
$$

${ }_{187}$ The absolute value of the jacobian of this mapping is given by

$$
\left|\mathcal{J}_{N+1}\right|=\operatorname{Exp}\left[-\int_{t_{0}}^{t}\left(\mu_{a}(s)+\sum_{j=1}^{N} \sqrt{v_{j}} \phi_{j}(s) y_{j+1}\right) \mathrm{d} s\right] \neq 0
$$

that is non-zero because is defined by an exponential. Then, applying Th. 1 and using independence between random variable $x_{0}$ and random vector $\boldsymbol{\xi}_{N}$, one obtains the joint PDF of random 


$$
\begin{aligned}
f_{\mathbf{y}_{N+1}}\left(y_{1}, \ldots, y_{N+1}\right)= & f_{\xi_{N+1}}\left(y_{1} \operatorname{Exp}\left[-\int_{t_{0}}^{t}\left(\mu_{a}(s)+\sum_{j=1}^{N} \sqrt{v_{j}} \phi_{j}(s) y_{j+1}\right] \mathrm{d} s\right], y_{2}, \ldots, y_{N+1}\right) \\
& \times \operatorname{Exp}\left[-\int_{t_{0}}^{t}\left(\mu_{a}(s)+\sum_{j=1}^{N} \sqrt{v_{j}} \phi_{j}(s) y_{j+1}\right) \mathrm{d} s\right] \\
= & f_{0}\left(y_{1} \operatorname{Exp}\left[-\int_{t_{0}}^{t}\left(\mu_{a}(s)+\sum_{j=1}^{N} \sqrt{v_{j}} \phi_{j}(s) y_{j+1}\right) \mathrm{d} s\right] f_{\xi_{N}}\left(y_{2}, \ldots, y_{N+1}\right)\right. \\
& \times \operatorname{Exp}\left[-\int_{t_{0}}^{t}\left(\mu_{a}(s)+\sum_{j=1}^{N} \sqrt{v_{j}} \phi_{j}(s) y_{j+1}\right) \mathrm{d} s\right] .
\end{aligned}
$$

192

193

thus expression (9) writes

$$
f_{1}^{N}(x, t)=\int_{\mathbb{R}^{N}} f_{0}\left(x \mathrm{e}^{-K_{N}\left(t, \xi_{N}\right)}\right) f_{\xi_{N}}\left(\xi_{1}, \ldots, \xi_{N}\right) \mathrm{e}^{-K_{N}\left(t, \xi_{N}\right)} \mathrm{d} \xi_{N} \cdots \mathrm{d} \xi_{1} .
$$


Additionally, the following hypotheses will be assumed throughout in the subsequent development.

H3 : $f_{0}\left(x_{0}\right)$ is Lipschitz in $\mathbb{R}$, i.e., $\exists L_{f_{0}}:\left|f_{0}\left(x_{0,1}\right)-f\left(x_{0,2}\right)\right| \leq L_{f_{0}}\left|x_{0,1}-x_{0,2}\right|, \quad \forall x_{0,1}, x_{0,2} \in \mathbb{R}$, 206 and

SP $a(t, \omega)$ admits a Karhunen-Loève expansion of type (3), H4: such that there exists a positive constant $C>0$ such that $\mathbb{E}\left[\mathrm{e}^{-4 K_{N}\left(t, \xi_{N}(\omega)\right)}\right] \leq C$, for all positive integer $N$. Later we will show that this condition can be guaranteed in practice (see Remark 2).

Let $\epsilon>0, \mathcal{J} \subset \mathbb{R}$ bounded, $(x, t) \in \mathcal{J} \times\left[t_{0}, T\right]$ an arbitrary point and $N>M$ integers. Taking into account (11), below we show that $\left\{f_{1}^{N}(x, t): N \geq 1\right\}$ is a Cauchy sequence by using several bounds that will be justified later. 


$$
\begin{aligned}
& \left|f_{1}^{N}(x, t)-f_{1}^{M}(x, t)\right| \\
& =\mid \int_{\mathbb{R}^{N}} f_{0}\left(x \mathrm{e}^{-K_{N}\left(t, \xi_{N}\right)}\right) f_{\xi_{N}}\left(\xi_{1}, \ldots, \xi_{N}\right) \mathrm{e}^{-K_{N}\left(t, \xi_{N}\right)} \mathrm{d} \xi_{N} \cdots \mathrm{d} \xi_{1} \\
& -\int_{\mathbb{R}^{M}} f_{0}\left(x \mathrm{e}^{-K_{M}\left(t, \xi_{M}\right)}\right) f_{\xi_{M}}\left(\xi_{1}, \ldots, \xi_{M}\right) \mathrm{e}^{-K_{M}\left(t, \xi_{M}\right)} \mathrm{d} \xi_{M} \cdots \mathrm{d} \xi_{1} \mid \\
& \stackrel{(\mathrm{I})}{=}\left|\int_{\mathbb{R}^{N}}\left[f_{0}\left(x \mathrm{e}^{-K_{N}\left(t, \xi_{N}\right)}\right) \mathrm{e}^{-K_{N}\left(t, \xi_{N}\right)}-f_{0}\left(x \mathrm{e}^{-K_{M}\left(t, \xi_{M}\right)}\right) \mathrm{e}^{-K_{M}\left(t, \xi_{M}\right)}\right] f_{\xi_{N}}\left(\xi_{1}, \ldots, \xi_{N}\right) \mathrm{d} \xi_{N} \cdots \mathrm{d} \xi_{1}\right| \\
& \leq \int_{\mathbb{R}^{N}}\left|\left[f_{0}\left(x \mathrm{e}^{-K_{N}\left(t, \xi_{N}\right)}\right) \mathrm{e}^{-K_{N}\left(t, \xi_{N}\right)}-f_{0}\left(x \mathrm{e}^{-K_{M}\left(t, \xi_{M}\right)}\right) \mathrm{e}^{-K_{M}\left(t, \xi_{M}\right)}\right]\right| f_{\xi_{N}}\left(\xi_{1}, \ldots, \xi_{N}\right) \mathrm{d} \xi_{N} \cdots \mathrm{d} \xi_{1} \\
& =\int_{\mathbb{R}^{N}} \mid\left[f_{0}\left(x \mathrm{e}^{-K_{N}\left(t, \boldsymbol{\xi}_{N}\right)}\right) \mathrm{e}^{-K_{N}\left(t, \boldsymbol{\xi}_{N}\right)}-f_{0}\left(x \mathrm{e}^{-K_{N}\left(t, \xi_{N}\right)}\right) \mathrm{e}^{-K_{M}\left(t, \xi_{M}\right)}\right. \\
& \left.+f_{0}\left(x \mathrm{e}^{-K_{N}\left(t, \xi_{N}\right)}\right) \mathrm{e}^{-K_{M}\left(t, \xi_{M}\right)}-f_{0}\left(x \mathrm{e}^{-K_{M}\left(t, \xi_{M}\right)}\right) \mathrm{e}^{-K_{M}\left(t, \xi_{M}\right)}\right] \mid f_{\xi_{N}}\left(\xi_{1}, \ldots, \xi_{N}\right) \mathrm{d} \xi_{N} \cdots \mathrm{d} \xi_{1} \\
& \leq \int_{\mathbb{R}^{N}}\left[f_{0}\left(x \mathrm{e}^{-K_{N}\left(t, \xi_{N}\right)}\right)\left|\mathrm{e}^{-K_{N}\left(t, \xi_{N}\right)}-\mathrm{e}^{-K_{M}\left(t, \xi_{M}\right)}\right|\right. \\
& \left.+\left|f_{0}\left(x \mathrm{e}^{-K_{N}\left(t, \xi_{N}\right)}\right)-f_{0}\left(x \mathrm{e}^{-K_{M}\left(t, \xi_{M}\right)}\right)\right| \mathrm{e}^{-K_{M}\left(t, \xi_{M}\right)}\right] f_{\xi_{N}}\left(\xi_{1}, \ldots, \xi_{N}\right) \mathrm{d} \xi_{N} \cdots \mathrm{d} \xi_{1} \\
& =\int_{\mathbb{R}^{N}} \underbrace{f_{0}\left(x \mathrm{e}^{-K_{N}\left(t, \xi_{N}\right)}\right)}_{(1)} \underbrace{\mathrm{e}^{-K_{N}\left(t, \xi_{N}\right)}-\mathrm{e}^{-K_{M}\left(t, \xi_{M}\right)}}_{(2)} \mid f_{\xi_{N}}\left(\xi_{1}, \ldots, \xi_{N}\right) \mathrm{d} \xi_{N} \cdots \mathrm{d} \xi_{1} \\
& +\int_{\mathbb{R}^{N}} \underbrace{\left|f_{0}\left(x \mathrm{e}^{-K_{N}\left(t, \xi_{N}\right)}\right)-f_{0}\left(x \mathrm{e}^{-K_{M}\left(t, \xi_{M}\right)}\right)\right|}_{(3)} \mathrm{e}^{-K_{M}\left(t, \xi_{M}\right)} f_{\xi_{N}}\left(\xi_{1}, \ldots, \xi_{N}\right) \mathrm{d} \xi_{N} \cdots \mathrm{d} \xi_{1} \\
& \stackrel{\text { (II) }}{<} L_{f_{0}}|x| \int_{\mathbb{R}^{N}}\left(\mathrm{e}^{-2 K_{N}\left(t, \xi_{N}\right)}+\mathrm{e}^{-K_{N}\left(t, \xi_{N}\right)-K_{M}\left(t, \xi_{M}\right)}\right)\left|K_{N}\left(t, \boldsymbol{\xi}_{N}\right)-K_{M}\left(t, \boldsymbol{\xi}_{M}\right)\right| f_{\xi_{N}}\left(\xi_{1}, \ldots, \xi_{N}\right) \mathrm{d} \xi_{N} \cdots \mathrm{d} \xi_{1} \\
& +F_{0} \int_{\mathbb{R}^{N}}\left(\mathrm{e}^{-K_{N}\left(t, \xi_{N}\right)}+\mathrm{e}^{-K_{M}\left(t, \xi_{M}\right)}\right)\left|K_{N}\left(t, \boldsymbol{\xi}_{N}\right)-K_{M}\left(t, \boldsymbol{\xi}_{M}\right)\right| f_{\xi_{N}}\left(\xi_{1}, \ldots, \xi_{N}\right) \mathrm{d} \xi_{N} \cdots \mathrm{d} \xi_{1} \\
& +L_{f_{0}}|x| \int_{\mathbb{R}^{N}}\left(\mathrm{e}^{-2 K_{M}\left(t, \xi_{M}\right)}+\mathrm{e}^{-K_{N}\left(t, \boldsymbol{\xi}_{N}\right)-K_{M}\left(t, \boldsymbol{\xi}_{M}\right)}\right)\left|K_{N}\left(t, \boldsymbol{\xi}_{N}\right)-K_{M}\left(t, \boldsymbol{\xi}_{M}\right)\right| f_{\xi_{N}}\left(\xi_{1}, \ldots, \xi_{N}\right) \mathrm{d} \xi_{N} \cdots \mathrm{d} \xi_{1} \\
& =L_{f_{0}}|x| \mathbb{E}\left[\left(\mathrm{e}^{-2 K_{N}\left(t, \boldsymbol{\xi}_{N}(\omega)\right)}+\mathrm{e}^{-K_{N}\left(t, \xi_{N}(\omega)\right)-K_{M}\left(t, \xi_{M}(\omega)\right)}\right)\left|K_{N}\left(t, \boldsymbol{\xi}_{N}(\omega)\right)-K_{M}\left(t, \boldsymbol{\xi}_{M}(\omega)\right)\right|\right] \\
& +F_{0} \mathbb{E}\left[\left(\mathrm{e}^{-K_{N}\left(t, \xi_{N}(\omega)\right)}+\mathrm{e}^{-K_{M}\left(t, \xi_{M}(\omega)\right)}\right)\left|K_{N}\left(t, \boldsymbol{\xi}_{N}(\omega)\right)-K_{M}\left(t, \boldsymbol{\xi}_{M}(\omega)\right)\right|\right] \\
& +L_{f_{0}}|x| \mathbb{E}\left[\left(\mathrm{e}^{-2 K_{M}\left(t, \xi_{M}(\omega)\right)}+\mathrm{e}^{-K_{N}\left(t, \xi_{N}(\omega)\right)-K_{M}\left(t, \xi_{M}(\omega)\right)}\right)\left|K_{N}\left(t, \boldsymbol{\xi}_{N}(\omega)\right)-K_{M}\left(t, \boldsymbol{\xi}_{M}(\omega)\right)\right|\right] .
\end{aligned}
$$




$$
\begin{aligned}
& \mid \int_{\mathbb{R}^{N}} f_{0}\left(x \mathrm{e}^{-K_{N}\left(t, \xi_{N}\right)}\right) f_{\xi_{N}}\left(\xi_{1}, \ldots, \xi_{N}\right) \mathrm{e}^{-K_{N}\left(t, \xi_{N}\right)} \mathrm{d} \xi_{N} \cdots \mathrm{d} \xi_{1} \\
& -\int_{\mathbb{R}^{M}} f_{0}\left(x \mathrm{e}^{-K_{M}\left(t, \xi_{M}\right)}\right) f_{\xi_{M}}\left(\xi_{1}, \ldots, \xi_{M}\right) \mathrm{e}^{-K_{M}\left(t, \xi_{M}\right)} \mathrm{d} \xi_{M} \cdots \mathrm{d} \xi_{1} \mid \\
& =\mid \int_{\mathbb{R}^{N}} f_{0}\left(x \mathrm{e}^{-K_{N}\left(t, \xi_{N}\right)}\right) f_{\xi_{N}}\left(\xi_{1}, \ldots, \xi_{N}\right) \mathrm{e}^{-K_{N}\left(t, \xi_{N}\right)} \mathrm{d} \xi_{N} \cdots \mathrm{d} \xi_{1} \\
& -\int_{\mathbb{R}^{M}} f_{0}\left(x \mathrm{e}^{-K_{M}\left(t, \xi_{M}\right)}\right)\left(\int_{\mathbb{R}^{N-M}} f_{\xi_{N}}\left(\xi_{1}, \ldots, \xi_{N}\right) \mathrm{d} \xi_{N} \cdots \mathrm{d} \xi_{M+1}\right) \mathrm{e}^{-K_{M}\left(t, \xi_{M}\right)} \mathrm{d} \xi_{M} \cdots \mathrm{d} \xi_{1} \mid \\
& =\mid \int_{\mathbb{R}^{N}} f_{0}\left(x \mathrm{e}^{-K_{N}\left(t, \xi_{N}\right)}\right) f_{\xi_{N}}\left(\xi_{1}, \ldots, \xi_{N}\right) \mathrm{e}^{-K_{N}\left(t, \xi_{N}\right)} \mathrm{d} \xi_{N} \cdots \mathrm{d} \xi_{1} \\
& -\int_{\mathbb{R}^{N}} f_{0}\left(x \mathrm{e}^{-K_{M}\left(t, \xi_{M}\right)}\right) f_{\xi_{N}}\left(\xi_{1}, \ldots, \xi_{N}\right) \mathrm{e}^{-K_{M}\left(t, \xi_{M}\right)} \mathrm{d} \xi_{N} \cdots \mathrm{d} \xi_{1} \mid \\
& =\left|\int_{\mathbb{R}^{N}}\left[f_{0}\left(x \mathrm{e}^{-K_{N}\left(t, \xi_{N}\right)}\right) f_{\xi_{N}}\left(\xi_{1}, \ldots, \xi_{N}\right) \mathrm{e}^{-K_{N}\left(t, \xi_{N}\right)}-f_{0}\left(x \mathrm{e}^{-K_{M}\left(t, \xi_{M}\right)}\right) f_{\xi_{N}}\left(\xi_{1}, \ldots, \xi_{N}\right) \mathrm{e}^{-K_{M}\left(t, \xi_{M}\right)}\right] \mathrm{d} \xi_{N} \cdots \mathrm{d} \xi_{1}\right| \\
& =\left|\int_{\mathbb{R}^{N}}\left[f_{0}\left(x \mathrm{e}^{-K_{N}\left(t, \xi_{N}\right)}\right) \mathrm{e}^{-K_{N}\left(t, \xi_{N}\right)}-f_{0}\left(x \mathrm{e}^{-K_{M}\left(t, \xi_{M}\right)}\right) \mathrm{e}^{-K_{M}\left(t, \xi_{M}\right)}\right] f_{\xi_{N}}\left(\xi_{1}, \ldots, \xi_{N}\right) \mathrm{d} \xi_{N} \cdots \mathrm{d} \xi_{1}\right|,
\end{aligned}
$$


which is just the right-hand side of (I). This justifies Step (I).

Step (II): Now we will legitimate bounds used in this step. Without loss of generality, let $F_{0}=f_{0}(0)$ and then we first bound the term (1) using hypothesis $\mathrm{H} 3$ :

$$
f_{0}\left(x \mathrm{e}^{-K_{N}\left(t, \xi_{N}(\omega)\right)}\right) \leq\left|f_{0}\left(x \mathrm{e}^{-K_{N}\left(t, \xi_{N}(\omega)\right)}\right)-f_{0}(0)\right|+\left|f_{0}(0)\right| \leq L_{f_{0}}|x| \mathrm{e}^{-K_{N}\left(t, \xi_{N}(\omega)\right)}+F_{0} .
$$

24 Secondly, we will obtain a bound for the product of the terms (1) and (2) as follows

$$
\begin{aligned}
f_{0}\left(x \mathrm{e}^{-K_{N}\left(t, \xi_{N}\right)}\right)\left|\mathrm{e}^{-K_{N}\left(t, \xi_{N}\right)}-\mathrm{e}^{-K_{M}\left(t, \xi_{M}\right)}\right| & \leq\left(L_{f_{0}}|x| \mathrm{e}^{-K_{N}\left(t, \xi_{N}(\omega)\right)}+F_{0}\right)\left|\mathrm{e}^{-K_{N}\left(t, \xi_{N}\right)}-\mathrm{e}^{-K_{M}\left(t, \xi_{M}\right)}\right| \\
& =L_{f_{0}}|x|\left|\mathrm{e}^{-2 K_{N}\left(t, \xi_{N}\right)}-\mathrm{e}^{-K_{N}\left(t, \xi_{N}\right)-K_{M}\left(t, \xi_{M}\right)}\right| \\
& +F_{0}\left|\mathrm{e}^{-K_{N}\left(t, \xi_{N}\right)}-\mathrm{e}^{-K_{M}\left(t, \xi_{M}\right)}\right| .
\end{aligned}
$$

225 Now, by applying the Mean Value Theorem twice to function $\mathrm{e}^{-z}$, it is guaranteed that

$$
\begin{gathered}
\left.\exists \delta_{t, \xi_{N}}^{(1)} \in\right] \min \left\{2 K_{N}\left(t, \boldsymbol{\xi}_{N}\right), K_{N}\left(t, \boldsymbol{\xi}_{N}\right)+K_{M}\left(t, \boldsymbol{\xi}_{M}\right)\right\}, \max \left\{2 K_{N}\left(t, \boldsymbol{\xi}_{N}\right), K_{N}\left(t, \boldsymbol{\xi}_{N}\right)+K_{M}\left(t, \boldsymbol{\xi}_{M}\right)\right\}[ \\
\text { such that : }\left|\mathrm{e}^{-2 K_{N}\left(t, \xi_{N}\right)}-\mathrm{e}^{-K_{N}\left(t, \xi_{M}\right)-K_{M}\left(t, \xi_{M}\right)}\right|=\mathrm{e}^{-\delta_{t, \xi_{N}}^{(1)}}\left|K_{N}\left(t, \boldsymbol{\xi}_{N}\right)-K_{M}\left(t, \boldsymbol{\xi}_{M}\right)\right|,
\end{gathered}
$$

226 and

$$
\begin{aligned}
& \left.\exists \delta_{t, \xi_{N}}^{(2)} \in\right] \min \left\{K_{N}\left(t, \boldsymbol{\xi}_{N}\right), K_{M}\left(t, \boldsymbol{\xi}_{M}\right)\right\}, \max \left\{K_{N}\left(t, \boldsymbol{\xi}_{N}\right), K_{M}\left(t, \boldsymbol{\xi}_{M}\right)\right\}[ \\
& \text { such that : }\left|\mathrm{e}^{-K_{N}\left(t, \xi_{N}\right)}-\mathrm{e}^{-K_{M}\left(t, \boldsymbol{\xi}_{M}\right)}\right|=\mathrm{e}^{-\delta_{t, \xi_{N}}^{(2)}}\left|K_{N}\left(t, \boldsymbol{\xi}_{N}\right)-K_{M}\left(t, \boldsymbol{\xi}_{M}\right)\right|,
\end{aligned}
$$

respectively. As a consequence of (15) and (16), one gets

$$
\mathrm{e}^{-\max \left\{2 K_{N}\left(t, \xi_{N}\right), K_{N}\left(t, \xi_{N}\right)+K_{M}\left(t, \xi_{M}\right)\right\}}<\mathrm{e}^{-\delta_{t, \xi_{N}}^{(1)}}<\mathrm{e}^{-\min \left\{2 K_{N}\left(t, \xi_{N}\right), K_{N}\left(t, \xi_{N}\right)+K_{M}\left(t, \xi_{M}\right)\right\}}
$$

228 and

$$
\mathrm{e}^{-\max \left\{K_{N}\left(t, \xi_{N}\right), K_{M}\left(t, \xi_{M}\right)\right\}}<\mathrm{e}^{-\delta_{t, \xi_{N}}^{(2)}}<\mathrm{e}^{-\min \left\{K_{N}\left(t, \xi_{N}\right), K_{M}\left(t, \xi_{M}\right)\right\}},
$$

respectively. Therefore,

$$
\mathrm{e}^{-\delta_{t, \xi_{N}}^{(1)}}<\mathrm{e}^{-2 K_{N}\left(t, \xi_{N}\right)}+\mathrm{e}^{-K_{N}\left(t, \xi_{N}\right)-K_{M}\left(t, \xi_{M}\right)}
$$

230 and

$$
\mathrm{e}^{-\delta_{t, \xi_{N}}^{(2)}}<\mathrm{e}^{-K_{N}\left(t, \xi_{N}\right)}+\mathrm{e}^{-K_{M}\left(t, \xi_{M}\right)},
$$

${ }_{231}$ respectively. Applying (15)-(16) and (19)-(20) in (14) one deduces

$$
\begin{aligned}
f_{0}\left(x \mathrm{e}^{-K_{N}\left(t, \boldsymbol{\xi}_{N}\right)}\right)\left|\mathrm{e}^{-K_{N}\left(t, \boldsymbol{\xi}_{N}\right)}-\mathrm{e}^{-K_{M}\left(t, \boldsymbol{\xi}_{M}\right)}\right| \leq & L_{f_{0}}|x|\left|\mathrm{e}^{-2 K_{N}\left(t, \boldsymbol{\xi}_{N}\right)}-\mathrm{e}^{-K_{N}\left(t, \boldsymbol{\xi}_{N}\right)-K_{M}\left(t, \boldsymbol{\xi}_{M}\right)}\right| \\
& +F_{0}\left|\mathrm{e}^{-K_{N}\left(t, \boldsymbol{\xi}_{N}\right)}-\mathrm{e}^{-K_{M}\left(t, \boldsymbol{\xi}_{M}\right)}\right| \\
& =L_{f_{0}}|x| \mathrm{e}^{-\delta_{t, \xi_{N}}^{(1)}}\left|K_{N}\left(t, \boldsymbol{\xi}_{N}\right)-K_{M}\left(t, \boldsymbol{\xi}_{M}\right)\right| \\
& +F_{0} \mathrm{e}^{-\delta_{t, \xi_{N}}^{(2)}}\left|K_{N}\left(t, \boldsymbol{\xi}_{N}\right)-K_{M}\left(t, \boldsymbol{\xi}_{M}\right)\right| \\
& <\left\{L_{f_{0}}|x|\left(\mathrm{e}^{-2 K_{N}\left(t, \boldsymbol{\xi}_{N}\right)}+\mathrm{e}^{-K_{N}\left(t, \boldsymbol{\xi}_{N}\right)-K_{M}\left(t, \xi_{M}\right)}\right)\right. \\
& \left.+F_{0}\left(\mathrm{e}^{-K_{N}\left(t, \boldsymbol{\xi}_{N}\right)}+\mathrm{e}^{-K_{M}\left(t, \xi_{M}\right)}\right)\right\}\left|K_{N}\left(t, \boldsymbol{\xi}_{N}\right)-K_{M}\left(t, \boldsymbol{\xi}_{M}\right)\right| .
\end{aligned}
$$


Now, we construct a bound for term (3) following
the one hand, by applying hypothesis $\mathrm{H} 3$ one gets

$$
\left|f_{0}\left(x \mathrm{e}^{-K_{N}\left(t, \xi_{N}\right)}\right)-f_{0}\left(x \mathrm{e}^{-K_{M}\left(t, \xi_{M}\right)}\right)\right| \leq L_{f_{0}}|x|\left|\mathrm{e}^{-K_{N}\left(t, \xi_{N}\right)}-\mathrm{e}^{-K_{M}\left(t, \xi_{M}\right)}\right| .
$$

${ }_{234}$ On the other hand, by applying the Mean Value Theorem to function $\mathrm{e}^{-z}$, it is guaranteed that

$$
\begin{aligned}
\exists \delta_{t, \boldsymbol{\xi}_{N}}^{(3)} \in & ] \min \left\{2 K_{M}\left(t, \boldsymbol{\xi}_{M}\right), K_{N}\left(t, \boldsymbol{\xi}_{N}\right)+K_{M}\left(t, \boldsymbol{\xi}_{M}\right)\right\}, \max \left\{2 K_{M}\left(t, \boldsymbol{\xi}_{M}\right), K_{N}\left(t, \boldsymbol{\xi}_{N}\right)+K_{M}\left(t, \boldsymbol{\xi}_{M}\right)\right\}[ \\
& \text { such that : }\left|\mathrm{e}^{-K_{N}\left(t, \boldsymbol{\xi}_{N}\right)-K_{M}\left(t, \boldsymbol{\xi}_{M}\right)}-\mathrm{e}^{-2 K_{M}\left(t, \boldsymbol{\xi}_{M}\right)}\right|=\mathrm{e}^{-\delta_{t, \xi_{N}}^{(3)}}\left|K_{N}\left(t, \boldsymbol{\xi}_{N}\right)-K_{M}\left(t, \boldsymbol{\xi}_{M}\right)\right| .
\end{aligned}
$$

235 Therefore,

$$
\mathrm{e}^{-\max \left\{2 K_{M}\left(t, \boldsymbol{\xi}_{M}\right), K_{N}\left(t, \boldsymbol{\xi}_{N}\right)+K_{M}\left(t, \boldsymbol{\xi}_{M}\right)\right\}}<\mathrm{e}^{-\delta_{t, \xi_{N}}^{(3)}}<\mathrm{e}^{-\min \left\{2 K_{M}\left(t, \boldsymbol{\xi}_{M}\right), K_{N}\left(t, \boldsymbol{\xi}_{N}\right)+K_{M}\left(t, \boldsymbol{\xi}_{M}\right)\right\}}
$$

236 and hence

$$
\mathrm{e}^{-\delta_{t, \xi_{N}}^{(3)}}<\mathrm{e}^{-2 K_{M}\left(t, \xi_{M}\right)}+\mathrm{e}^{-K_{N}\left(t, \xi_{N}\right)-K_{M}\left(t, \boldsymbol{\xi}_{M}\right)} .
$$

237 Multiplying (21) by $\mathrm{e}^{-K_{M}\left(t, \xi_{M}\right)}$ and applying (22) and (24), one deduces

$$
\begin{aligned}
\left|f_{0}\left(x \mathrm{e}^{-K_{N}\left(t, \xi_{N}\right)}\right)-f_{0}\left(x \mathrm{e}^{-K_{M}\left(t, \xi_{M}\right)}\right)\right| \mathrm{e}^{-K_{M}\left(t, \boldsymbol{\xi}_{M}\right)} & \leq L_{f_{0}}|x|\left|\mathrm{e}^{-K_{N}\left(t, \boldsymbol{\xi}_{N}\right)}-\mathrm{e}^{-K_{M}\left(t, \boldsymbol{\xi}_{M}\right)}\right| \mathrm{e}^{-K_{M}\left(t, \xi_{M}\right)} \\
& =L_{f_{0}}|x|\left|\mathrm{e}^{-K_{N}\left(t, \boldsymbol{\xi}_{N}\right)-K_{M}\left(t, \boldsymbol{\xi}_{M}\right)}-\mathrm{e}^{-2 K_{M}\left(t, \boldsymbol{\xi}_{M}\right)}\right| \\
& =L_{f_{0}}|x| \mathrm{e}^{-\delta_{t, \xi_{N}}^{(3)}}\left|K_{N}\left(t, \boldsymbol{\xi}_{N}\right)-K_{M}\left(t, \boldsymbol{\xi}_{M}\right)\right| \\
& <L_{f_{0}}|x|\left(\mathrm{e}^{-2 K_{M}\left(t, \boldsymbol{\xi}_{M}\right)}+\mathrm{e}^{-K_{N}\left(t, \boldsymbol{\xi}_{N}\right)-K_{M}\left(t, \boldsymbol{\xi}_{M}\right)}\right)\left|K_{N}\left(t, \boldsymbol{\xi}_{N}\right)-K_{M}\left(t, \boldsymbol{\xi}_{M}\right)\right| .
\end{aligned}
$$

${ }_{238}$ Now, we will obtain bound for every expectation appearing in (12). To this end, we apply 
239

Cauchy-Schwarz inequality for expectations

$$
\begin{aligned}
& \mathbb{E}\left[\left(\mathrm{e}^{-2 K_{N}\left(t, \boldsymbol{\xi}_{N}(\omega)\right)}+\mathrm{e}^{-K_{N}\left(t, \boldsymbol{\xi}_{N}(\omega)\right)-K_{M}\left(t, \boldsymbol{\xi}_{M}(\omega)\right)}\right)\left|K_{N}\left(t, \boldsymbol{\xi}_{N}(\omega)\right)-K_{M}\left(t, \boldsymbol{\xi}_{M}(\omega)\right)\right|\right] \\
= & \mathbb{E}\left[\mathrm{e}^{-2 K_{N}\left(t, \boldsymbol{\xi}_{N}(\omega)\right)}\left|K_{N}\left(t, \boldsymbol{\xi}_{N}(\omega)\right)-K_{M}\left(t, \boldsymbol{\xi}_{M}(\omega)\right)\right|\right] \\
+ & \mathbb{E}\left[\mathrm{e}^{-K_{N}\left(t, \boldsymbol{\xi}_{N}(\omega)\right)-K_{M}\left(t, \boldsymbol{\xi}_{M}(\omega)\right)}\left|K_{N}\left(t, \boldsymbol{\xi}_{N}(\omega)\right)-K_{M}\left(t, \boldsymbol{\xi}_{M}(\omega)\right)\right|\right] \\
\leq & \left(\mathbb{E}\left[\mathrm{e}^{-4 K_{N}\left(t, \boldsymbol{\xi}_{N}(\omega)\right)}\right]\right)^{1 / 2}\left(\mathbb{E}\left[\left|K_{N}\left(t, \boldsymbol{\xi}_{N}(\omega)\right)-K_{M}\left(t, \boldsymbol{\xi}_{M}(\omega)\right)\right|^{2}\right]\right)^{1 / 2} \\
+ & \left(\mathbb{E}\left[\mathrm{e}^{-2 K_{N}\left(t, \boldsymbol{\xi}_{N}(\omega)\right)} \mathrm{e}^{-2 K_{M}\left(t, \boldsymbol{\xi}_{M}(\omega)\right)}\right]\right)^{1 / 2}\left(\mathbb{E}\left[\left|K_{N}\left(t, \boldsymbol{\xi}_{N}(\omega)\right)-K_{M}\left(t, \boldsymbol{\xi}_{M}(\omega)\right)\right|^{2}\right]\right)^{1 / 2} \\
\leq & \left(\mathbb{E}\left[\mathrm{e}^{-4 K_{N}\left(t, \boldsymbol{\xi}_{N}(\omega)\right)}\right]\right)^{1 / 2}\left(\mathbb{E}\left[\left|K_{N}\left(t, \boldsymbol{\xi}_{N}(\omega)\right)-K_{M}\left(t, \boldsymbol{\xi}_{M}(\omega)\right)\right|^{2}\right]\right)^{1 / 2} \\
+ & \left(\mathbb{E}\left[\mathrm{e}^{-4 K_{N}\left(t, \boldsymbol{\xi}_{N}(\omega)\right)}\right]\right)^{1 / 4}\left(\mathbb{E}\left[\mathrm{e}^{-4 K_{M}\left(t, \boldsymbol{\xi}_{M}(\omega)\right)}\right]\right)^{1 / 4}\left(\mathbb{E}\left[\left|K_{N}\left(t, \boldsymbol{\xi}_{N}(\omega)\right)-K_{M}\left(t, \boldsymbol{\xi}_{M}(\omega)\right)\right|^{2}\right]\right)^{1 / 2} \\
= & \left\{\left(\mathbb{E}\left[\mathrm{e}^{-4 K_{N}\left(t, \boldsymbol{\xi}_{N}(\omega)\right)}\right]\right)^{1 / 2}+\left(\mathbb{E}\left[\mathrm{e}^{-4 K_{N}\left(t, \xi_{N}(\omega)\right)}\right]\right)^{1 / 4}\left(\mathbb{E}\left[\mathrm{e}^{-4 K_{M}\left(t, \xi_{M}(\omega)\right)}\right]\right)^{1 / 4}\right\} \\
\times & \left(\mathbb{E}\left[\left|K_{N}\left(t, \boldsymbol{\xi}_{N}(\omega)\right)-K_{M}\left(t, \boldsymbol{\xi}_{M}(\omega)\right)\right|^{2}\right]\right)^{1 / 2},
\end{aligned}
$$

240

14 


$$
\begin{aligned}
& \mathbb{E}\left[\left(\mathrm{e}^{-K_{N}\left(t, \boldsymbol{\xi}_{N}(\omega)\right)}+\mathrm{e}^{-K_{M}\left(t, \boldsymbol{\xi}_{M}(\omega)\right)}\right)\left|K_{N}\left(t, \boldsymbol{\xi}_{N}(\omega)\right)-K_{M}\left(t, \boldsymbol{\xi}_{M}(\omega)\right)\right|\right] \\
&= \mathbb{E}\left[\mathrm{e}^{-K_{N}\left(t, \boldsymbol{\xi}_{N}(\omega)\right)}\left|K_{N}\left(t, \boldsymbol{\xi}_{N}(\omega)\right)-K_{M}\left(t, \boldsymbol{\xi}_{M}(\omega)\right)\right|\right] \\
&+ \mathbb{E}\left[\mathrm{e}^{-K_{M}\left(t, \boldsymbol{\xi}_{M}(\omega)\right)}\left|K_{N}\left(t, \boldsymbol{\xi}_{N}(\omega)\right)-K_{M}\left(t, \boldsymbol{\xi}_{M}(\omega)\right)\right|\right] \\
& \leq\left(\mathbb{E}\left[\mathrm{e}^{-2 K_{N}\left(t, \boldsymbol{\xi}_{N}(\omega)\right)}\right]\right)^{1 / 2}\left(\mathbb{E}\left[\left|K_{N}\left(t, \boldsymbol{\xi}_{N}(\omega)\right)-K_{M}\left(t, \boldsymbol{\xi}_{M}(\omega)\right)\right|^{2}\right]\right)^{1 / 2} \\
&+\left(\mathbb{E}\left[\mathrm{e}^{-2 K_{M}\left(t, \xi_{M}(\omega)\right)}\right]\right)^{1 / 2}\left(\mathbb{E}\left[\left|K_{N}\left(t, \boldsymbol{\xi}_{N}(\omega)\right)-K_{M}\left(t, \boldsymbol{\xi}_{M}(\omega)\right)\right|^{2}\right]\right)^{1 / 2} \\
& \leq\left(\mathbb{E}\left[\mathrm{e}^{-4 K_{N}\left(t, \boldsymbol{\xi}_{N}(\omega)\right)}\right]\right)^{1 / 4}\left(\mathbb{E}\left[\left|K_{N}\left(t, \boldsymbol{\xi}_{N}(\omega)\right)-K_{M}\left(t, \boldsymbol{\xi}_{M}(\omega)\right)\right|^{2}\right]\right)^{1 / 2} \\
&+\left(\mathbb{E}\left[\mathrm{e}^{-4 K_{M}\left(t, \xi_{M}(\omega)\right)}\right]\right)^{1 / 4}\left(\mathbb{E}\left[\left|K_{N}\left(t, \boldsymbol{\xi}_{N}(\omega)\right)-K_{M}\left(t, \boldsymbol{\xi}_{M}(\omega)\right)\right|^{2}\right]\right)^{1 / 2} \\
&=\left\{\left(\mathbb{E}\left[\mathrm{e}^{-4 K_{N}\left(t, \boldsymbol{\xi}_{N}(\omega)\right)}\right]\right)^{1 / 4}+\left(\mathbb{E}\left[\mathrm{e}^{-4 K_{M}\left(t, \boldsymbol{\xi}_{M}(\omega)\right)}\right]\right)^{1 / 4}\right\} \\
& \times\left(\mathbb{E}\left[\left|K_{N}\left(t, \boldsymbol{\xi}_{N}(\omega)\right)-K_{M}\left(t, \boldsymbol{\xi}_{M}(\omega)\right)\right|^{2}\right]\right)^{1 / 2},
\end{aligned}
$$


242 and

$$
\begin{aligned}
& \mathbb{E}\left[\left(\mathrm{e}^{-2 K_{M}\left(t, \boldsymbol{\xi}_{M}(\omega)\right)}+\mathrm{e}^{-K_{N}\left(t, \boldsymbol{\xi}_{N}(\omega)\right)-K_{M}\left(t, \boldsymbol{\xi}_{M}(\omega)\right)}\right)\left|K_{N}\left(t, \boldsymbol{\xi}_{N}(\omega)\right)-K_{M}\left(t, \boldsymbol{\xi}_{M}(\omega)\right)\right|\right] \\
= & \mathbb{E}\left[\mathrm{e}^{-2 K_{M}\left(t, \boldsymbol{\xi}_{M}(\omega)\right)}\left|K_{N}\left(t, \boldsymbol{\xi}_{N}(\omega)\right)-K_{M}\left(t, \boldsymbol{\xi}_{M}(\omega)\right)\right|\right] \\
+ & \mathbb{E}\left[\mathrm{e}^{-K_{N}\left(t, \boldsymbol{\xi}_{N}(\omega)\right)-K_{M}\left(t, \xi_{M}(\omega)\right)}\left|K_{N}\left(t, \boldsymbol{\xi}_{N}(\omega)\right)-K_{M}\left(t, \boldsymbol{\xi}_{M}(\omega)\right)\right|\right] \\
\leq & \left(\mathbb{E}\left[\mathrm{e}^{-4 K_{M}\left(t, \boldsymbol{\xi}_{M}(\omega)\right)}\right]\right)^{1 / 2}\left(\mathbb{E}\left[\left|K_{N}\left(t, \boldsymbol{\xi}_{N}(\omega)\right)-K_{M}\left(t, \boldsymbol{\xi}_{M}(\omega)\right)\right|^{2}\right]\right)^{1 / 2} \\
+ & \left(\mathbb{E}\left[\mathrm{e}^{-2 K_{N}\left(t, \boldsymbol{\xi}_{N}(\omega)\right)} \mathrm{e}^{-2 K_{M}\left(t, \boldsymbol{\xi}_{M}\right)(\omega)}\right]\right)^{1 / 2}\left(\mathbb{E}\left[\left|K_{N}\left(t, \boldsymbol{\xi}_{N}(\omega)\right)-K_{M}\left(t, \boldsymbol{\xi}_{M}(\omega)\right)\right|^{2}\right]\right)^{1 / 2} \\
\leq & \left(\mathbb{E}\left[\mathrm{e}^{-4 K_{M}\left(t, \boldsymbol{\xi}_{M}(\omega)\right)}\right]\right)^{1 / 2}\left(\mathbb{E}\left[\left|K_{N}\left(t, \boldsymbol{\xi}_{N}(\omega)\right)-K_{M}\left(t, \boldsymbol{\xi}_{M}(\omega)\right)\right|^{2}\right]\right)^{1 / 2} \\
+ & \left(\mathbb{E}\left[\mathrm{e}^{-4 K_{N}\left(t, \boldsymbol{\xi}_{N}(\omega)\right)}\right]\right)^{1 / 4}\left(\mathbb{E}\left[\mathrm{e}^{-4 K_{M}\left(t, \boldsymbol{\xi}_{M}(\omega)\right)}\right]\right)^{1 / 4}\left(\mathbb{E}\left[\left|K_{N}\left(t, \boldsymbol{\xi}_{N}(\omega)\right)-K_{M}\left(t, \boldsymbol{\xi}_{M}(\omega)\right)\right|^{2}\right]\right)^{1 / 2} \\
\leq & \left\{\left(\mathbb{E}\left[\mathrm{e}^{-4 K_{M}\left(t, \boldsymbol{\xi}_{M}(\omega)\right)}\right]\right)^{1 / 2}+\left(\mathbb{E}\left[\mathrm{e}^{-4 K_{N}\left(t, \boldsymbol{\xi}_{N}(\omega)\right)}\right]\right)^{1 / 4}\left(\mathbb{E}\left[\mathrm{e}^{-4 K_{M}\left(t, \boldsymbol{\xi}_{M}(\omega)\right)}\right]\right)^{1 / 4}\right\} \\
\times & \left(\mathbb{E}\left[\left|K_{N}\left(t, \boldsymbol{\xi}_{N}(\omega)\right)-K_{M}\left(t, \boldsymbol{\xi}_{M}(\omega)\right)\right|^{2}\right]\right)^{1 / 2} \cdot
\end{aligned}
$$

243 By substituting the three bounds given in (26)-(28) into expression (12), we obtain

$$
\begin{aligned}
\left|f_{1}^{N}(x, t)-f_{1}^{M}(x, t)\right| & \leq\left[L_{f_{0}}|x|\left\{\left(\mathbb{E}\left[\mathrm{e}^{-4 K_{N}\left(t, \xi_{N}(\omega)\right)}\right]\right)^{1 / 2}+\left(\mathbb{E}\left[\mathrm{e}^{-4 K_{N}\left(t, \xi_{N}(\omega)\right)}\right]\right)^{1 / 4}\left(\mathbb{E}\left[\mathrm{e}^{-4 K_{M}\left(t, \xi_{M}(\omega)\right)}\right]\right)^{1 / 4}\right\}\right. \\
& +F_{0}\left\{\left(\mathbb{E}\left[\mathrm{e}^{-4 K_{N}\left(t, \xi_{N}(\omega)\right)}\right]\right)^{1 / 4}+\left(\mathbb{E}\left[\mathrm{e}^{-4 K_{M}\left(t, \xi_{M}(\omega)\right)}\right]\right)^{1 / 4}\right\} \\
& \left.+L_{f_{0}}|x|\left\{\left(\mathbb{E}\left[\mathrm{e}^{-4 K_{M}\left(t, \xi_{M}(\omega)\right)}\right]\right)^{1 / 2}+\left(\mathbb{E}\left[\mathrm{e}^{-4 K_{N}\left(t, \xi_{N}(\omega)\right)}\right]\right)^{1 / 4}\left(\mathbb{E}\left[\mathrm{e}^{-4 K_{M}\left(t, \xi_{M}(\omega)\right)}\right]\right)^{1 / 4}\right\}\right] \\
& \times\left(\mathbb{E}\left[\left|K_{N}\left(t, \boldsymbol{\xi}_{N}(\omega)\right)-K_{M}\left(t, \boldsymbol{\xi}_{M}(\omega)\right)\right|^{2}\right]\right)^{1 / 2} .
\end{aligned}
$$

244 Now, using hypothesis H4 one gets

$$
\begin{aligned}
\left|f_{1}^{N}(x, t)-f_{1}^{M}(x, t)\right| & \leq\left[L_{f_{0}}|x|\left\{C^{1 / 2}+C^{1 / 4} \times C^{1 / 4}\right\}+F_{0}\left\{C^{1 / 4}+C^{1 / 4}\right\}+L_{f_{0}}|x|\left\{C^{1 / 2}+C^{1 / 4} \times C^{1 / 4}\right\}\right] \\
& \times\left(\mathbb{E}\left[\left|K_{N}\left(t, \boldsymbol{\xi}_{N}(\omega)\right)-K_{M}\left(t, \boldsymbol{\xi}_{M}(\omega)\right)\right|^{2}\right]\right)^{1 / 2} \\
& =[\alpha|x|+\beta]\left(\mathbb{E}\left[\left|K_{N}\left(t, \boldsymbol{\xi}_{N}(\omega)\right)-K_{M}\left(t, \boldsymbol{\xi}_{M}(\omega)\right)\right|^{2}\right]\right)^{1 / 2},
\end{aligned}
$$

245 where

$$
\alpha=4 C^{1 / 2} L_{f_{0}}, \quad \beta=2 C^{1 / 4} F_{0} .
$$


Let us observe that,

$$
\begin{aligned}
\mathbb{E}\left[\left|K_{N}\left(t, \boldsymbol{\xi}_{N}(\omega)\right)-K_{M}\left(t, \boldsymbol{\xi}_{M}(\omega)\right)\right|^{2}\right] & =\mathbb{E}\left[\left(\int_{t_{0}}^{t} \sum_{j=M+1}^{N} \sqrt{v_{j}} \phi_{j}(s) \xi_{j}(\omega) \mathrm{d} s\right)^{2}\right] \\
& \leq \mathbb{E}\left[\left(\int_{t_{0}}^{t} 1^{2} \mathrm{~d} s\right)\left(\int_{t_{0}}^{t}\left(\sum_{j=M+1}^{N} \sqrt{v_{j}} \phi_{j}(s) \xi_{j}(\omega)\right)^{2} \mathrm{~d} s\right)\right] \\
& =\left(t-t_{0}\right) \mathbb{E}\left[\int_{t_{0}}^{t}\left(\sum_{j=M+1}^{N} \sqrt{v_{j}} \phi_{j}(s) \xi_{j}(\omega)\right)^{2} \mathrm{~d} s\right] \\
& \leq\left(T-t_{0}\right) \mathbb{E}\left[\int_{t_{0}}^{T}\left(\sum_{j=M+1}^{N} \sqrt{v_{j}} \phi_{j}(s) \xi_{j}(\omega)\right)^{2} \mathrm{~d} s\right] \\
& =\left(T-t_{0}\right) \mathbb{E}\left[\int_{t_{0}}^{T}\left(a_{N}(t, \omega)-a_{M}(t, \omega)\right)^{2} \mathrm{~d} s\right] \\
& =\left(T-t_{0}\right) \int_{t_{0}}^{T} \mathbb{E}\left[\left(a_{N}(t, \omega)-a_{M}(t, \omega)\right)^{2}\right] \mathrm{d} s \\
& =\left(T-t_{0}\right)\left(\left\|a_{N}-a_{M}\right\|_{\left.\mathrm{L}^{2}\left(\Omega, \mathrm{L}^{2}\left(I_{0}, T\right]\right)\right)}\right)^{2},
\end{aligned}
$$

where the first inequality has been obtained by applying the Cauchy-Schwarz inequality for integrals and the monotonicity of the expectation operator $\mathbb{E}[\cdot]$, and in the last step, we have used the definition of the norm $\|\cdot\|_{\mathrm{L}^{2}\left(\Omega, \mathrm{L}^{2}(\mathcal{T})\right)}$ for $\mathcal{T}=\left[t_{0}, T\right]$. Substituting this latter conclusion in (30) and applying the Cauchy convergence condition for the KLE of diffusion coefficient $a(t, \omega)$ in the norm $\|\cdot\|_{\mathrm{L}^{2}\left(\Omega, \mathrm{L}^{2}\left(\left[t_{0}, T\right]\right)\right)}$ (see Theorem 2$)$, one deduces

$$
\begin{aligned}
\left|f_{1}^{N}(x, t)-f_{1}^{M}(x, t)\right| & \leq[\alpha|x|+\beta]\left(\mathbb{E}\left[\left|K_{N}\left(t, \boldsymbol{\xi}_{N}(\omega)\right)-K_{M}\left(t, \boldsymbol{\xi}_{M}(\omega)\right)\right|^{2}\right]\right)^{1 / 2} \\
& \leq[\alpha|x|+\beta] \sqrt{T-t_{0}}\left\|a_{N}-a_{M}\right\|_{\mathrm{L}^{2}\left(\Omega, \mathrm{L}^{2}\left(\left[t_{0}, T\right]\right)\right)} \underset{N, M \rightarrow+\infty}{\longrightarrow} 0 .
\end{aligned}
$$

This proves that $\left\{f_{1}^{N} \equiv f_{1}^{N}(x, t): N \geq 1\right\}$ is a uniformly Cauchy sequence in $\mathcal{J} \times\left[t_{0}, T\right]$ for all $\mathcal{J} \subset \mathbb{R}$ bounded.

Summarizing, the following result has been established

Proposition 1. Under hypotheses H1-H4, the sequence $\left\{f_{1}^{N}(x, t): N \geq 1\right\}$ of 1-PDFs, defined by (9), converges uniformly in $(x, t) \in \mathcal{J} \times \mathcal{T}$ for all $\mathcal{J} \subset \mathbb{R}$ bounded, to the exact $1-P D F, f_{1}(x, t)$, of the solution SP of random IVP (2).

Remark 1. Here, we will show that hypothesis H3 is fulfilled by the PDF of a wide variety of RVs. In fact, as a consequence of the Mean Value Theorem it is well know that if a function, say $f_{0}$, has bounded first derivative in $\mathbb{R}$, then $f_{0}$ is Lipschitz over the whole real line. It is straightforwardly to check that the PDF of important RVs such as Uniform, Beta, Gaussian, Gamma, etc. have bounded first derivative over the whole real line. For example, if $f_{0}$ is the PDF of an Exponential RV of parameter $\lambda>0$, then $f_{0}\left(x_{0}\right)=\lambda \mathrm{e}^{-\lambda x_{0}}, x_{0}, \lambda>0$. As $\frac{\mathrm{d} f_{0}}{\mathrm{~d} x_{0}}=-\lambda^{2} \mathrm{e}^{-\lambda x_{0}}$ is continuous in $\left[0,+\infty\left[\right.\right.$ and $\lim _{x_{0} \rightarrow+\infty} \frac{\mathrm{d} f_{0}}{\mathrm{~d} x_{0}}=0$, therefore $f_{0}\left(x_{0}\right)$ is Lipschitz in $[0,+\infty[$. 


$$
\mathbb{E}\left[\mathrm{e}^{d K_{M}\left(t, \xi_{M}(\omega)\right)}\right] \leq \mathrm{e}^{d \int_{t_{0}}^{t} \mu_{a}(s) \mathrm{d} s} \mathrm{e}^{\frac{d^{2}}{2}\left(T-t_{0}\right) \sum_{j=1}^{M} v_{j} \int_{t_{0}}^{T}\left(\phi_{j}(s)\right)^{2} \mathrm{~d} s} \leq \mathrm{e}^{d \int_{t_{0}}^{t} \mu_{a}(s) \mathrm{d} s} \mathrm{e}^{\frac{d^{2}}{2}\left(T-t_{0}\right) \int_{t_{0}}^{T}\left(\sum_{j=1}^{\infty} v_{j}\left(\phi_{j}(s)\right)^{2}\right) \mathrm{d} s} .
$$

Remark 2. We will show that hypothesis $\mathrm{H} 4$ is not restrictive in practice. In fact, it is important to observe that given the coefficient $a(t, \omega) \in \mathrm{L}^{2}\left(\Omega, \mathrm{L}^{2}(\mathcal{T})\right)$ of the random IVP (2), then according to KLE, the involved RVs $\xi_{j}(\omega)$ can be chosen in many ways so that $\mathbb{E}\left[\xi_{j}(\omega)\right]=0, \mathbb{V}\left[\xi_{j}(\omega)\right]=1$ and they are uncorrelated $\left(\mathbb{E}\left[\xi_{i}(\omega) \xi_{j}(\omega)\right]=0\right.$ for $\left.i \neq j\right)$. As in our case they must be absolutely continuous RVs, we can choose them so that they are uncorrelated Gaussian RVs with zero mean and unit variance, $\xi_{j}(\omega) \sim \mathrm{N}(0 ; 1)$. Next, we prove that making this choice, then hypothesis $\mathrm{H} 4$ holds. First, observe that taking into account (10), the expectation involved in $\mathrm{H} 4$ can be written as

$$
\mathbb{E}\left[\mathrm{e}^{d K_{M}\left(t, \xi_{M}(\omega)\right)}\right]=\mathrm{e}^{d \int_{t_{0}}^{t} \mu_{a}(s) \mathrm{d} s} \mathbb{E}\left[\prod_{j=1}^{M} \mathrm{e}^{d \boldsymbol{\xi}_{j}(\omega) \sqrt{v_{j}} \int_{t_{0}}^{t} \phi_{j}(s) \mathrm{d} s}\right]=\mathrm{e}^{d \int_{t_{0}}^{t} \mu_{a}(s) \mathrm{d} s} \prod_{j=1}^{M} \mathbb{E}\left[\mathrm{e}^{d \boldsymbol{\xi}_{j}(\omega) \sqrt{v_{j}} \int_{t_{0}}^{t} \phi_{j}(s) \mathrm{d} s}\right],
$$

where in the last step we have used that $\xi_{j}(\omega)$ are independent RVs (since they are uncorrelated and Gaussian), hence the expectation of the product is the product of expectations. Now, we use the following property

$$
\mathbb{E}\left[\mathrm{e}^{\lambda Z(\omega)}\right]=\mathrm{e}^{\frac{\lambda^{2}}{2}}, \quad \lambda \in \mathbb{R}, Z(\omega) \sim \mathrm{N}(0 ; 1),
$$

o compute every factor of the last product. This leads to

$$
\mathbb{E}\left[\mathrm{e}^{d K_{M}\left(t, \xi_{M}(\omega)\right)}\right]=\mathrm{e}^{d \int_{t_{0}}^{t} \mu_{a}(s) \mathrm{d} s} \prod_{j=1}^{M} \mathrm{e}^{\frac{d^{2}}{2} v_{j}\left(\int_{t_{0}}^{t} \phi_{j}(s) \mathrm{d} s\right)^{2}}=\mathrm{e}^{d \int_{t_{0}}^{t} \mu_{a}(s) \mathrm{d} s} \mathrm{e}^{\frac{d^{2}}{2} \sum_{j=1}^{M} v_{j}\left(\int_{t_{0}}^{t} \phi_{j}(s) \mathrm{d} s\right)^{2}} .
$$

Applying the Cauchy-Schwarz inequality for integrals one gets

$$
\left(\int_{t_{0}}^{t} \phi_{j}(s) \mathrm{d} s\right)^{2} \leq\left(t-t_{0}\right)\left(\int_{t_{0}}^{t}\left(\phi_{j}(s)\right)^{2} \mathrm{~d} s\right) \leq\left(T-t_{0}\right)\left(\int_{t_{0}}^{T}\left(\phi_{j}(s)\right)^{2} \mathrm{~d} s\right), \quad t_{0} \leq t \leq T .
$$

Therefore, expression (33) can be bounded as follows

Now, let us bound every integral term in the right-hand side of this expression, thus proving the finiteness of $\mathbb{E}\left[\mathrm{e}^{d K_{M}\left(t, \xi_{M}(\omega)\right)}\right]<+\infty$. As a consequence, taking $d=-4$ we will have shown an important scenario where hypothesis $\mathrm{H} 4$ holds. On the one hand, by Cauchy-Schwarz inequality for integrals, using that $\left(\mu_{a}(s)\right)^{2}=\left(\mathbb{E}[(a(s)])^{2} \leq \mathbb{E}\left[(a(s))^{2}\right]\right.$ and the fact that $a \in \mathrm{L}^{2}\left(\Omega, \mathrm{L}^{2}\left(\left[t_{0}, T\right]\right)\right)$, one gets

$$
\begin{aligned}
\int_{t_{0}}^{t}\left|\mu_{a}(s)\right| \mathrm{d} s & \leq \int_{t_{0}}^{T}\left|\mu_{a}(s)\right| \mathrm{d} s \\
& \leq \sqrt{T-t_{0}}\left(\int_{t_{0}}^{t}\left(\mu_{a}(s)\right)^{2} \mathrm{~d} s\right)^{1 / 2} \\
& \leq \sqrt{T-t_{0}}\left(\int_{t_{0}}^{T} \mathbb{E}\left[(a(s))^{2}\right] \mathrm{d} s\right)^{1 / 2} \\
& =\sqrt{T-t_{0}}\|a\|_{\mathrm{L}^{2}\left(\Omega, \mathrm{L}^{2}\left(\left[t_{0}, T\right]\right)\right)}<+\infty .
\end{aligned}
$$


287 and

Since $\left|\int_{t_{0}}^{t} \mu_{a}(s) \mathrm{d} s\right| \leq \int_{t_{0}}^{t}\left|\mu_{a}(s)\right| \mathrm{d} s$, this justifies $\mathrm{e}^{b \int_{t_{0}}^{t} \mu_{a}(s) \mathrm{d} s}<+\infty$, which is the first factor of the right-hand side in (34). On the other hand, let us observe

$\mathbb{E}\left[(a(s))^{2}\right]=\left(\mu_{a}(s)\right)^{2}+\sum_{j=1}^{\infty} v_{j}\left(\phi_{j}(s)\right)^{2}, \quad a(s)=\mu_{a}(s)+\sum_{j=1}^{+\infty} \sqrt{v_{j}} \phi_{j}(s) \xi_{j}(\omega)$ in $\mathrm{L}^{2}\left(\Omega, \mathrm{L}^{2}\left(\left[t_{0}, T\right]\right)\right)$,

$$
\sum_{j=1}^{\infty} v_{j}\left(\phi_{j}(s)\right)^{2} \leq \mathbb{E}\left[(a(s))^{2}\right]
$$

$$
\int_{t_{0}}^{T} \sum_{j=1}^{\infty} v_{j}\left(\phi_{j}(s)\right)^{2} \mathrm{~d} s \leq \int_{t_{0}}^{T} \mathbb{E}\left[(a(s))^{2}\right] \mathrm{d} s=\left(\|a\|_{\mathrm{L}^{2}\left(\Omega, \mathrm{L}^{2}\left(\left[t_{0}, T\right]\right)\right)}\right)^{2}<+\infty .
$$

Therefore, the second factor of the right-hand side in (34) is finite, i.e. $\mathrm{e}^{\frac{b^{2}}{2}\left(T-t_{0}\right) \int_{t_{0}}^{T}\left(\sum_{j=1}^{\infty} v_{j}\left(\phi_{j}(s)\right)^{2}\right) \mathrm{d} s}<$ $+\infty$. Summarizing, if we choose $\xi_{j}(\omega)$ in the KLE as uncorrelated standard Gaussian RVs, then hypothesis H4 is guaranteed.

\section{Examples}

In this section, we will show two examples. In the first example, we will consider that the SP $a(t, \omega)$, playing the role of diffusion coefficient in the random IVP (2), is the so-called Brownian motion or standard Wiener process. As the exact distribution of the Brownian motion is known, then the exact 1-PDF $f_{1}(x, t)$ of the solution SP $x(t, \omega)$ to (2) can be derived. Hence, this first example will be used as a test to compare the approximations, $f_{1}^{N}(x, t)$ given by (9), for different values of the truncation order $N$ against the exact values. In the second example a covariance function is considered. Then, from the knowledge of its eigenpairs $\left\{\left(v_{j}, \phi_{j}(t)\right): j \geq\right.$ $1\}$, approximations of the 1-PDF are given. In both examples, approximations of the mean and standard deviation of $x(t, \omega)$ are given from $f_{1}^{N}(x, t)$. Finally, in both examples we provide error measures in order to quantify the accuracy of approximations of the 1-PDF, the mean and the standard deviation.

\subsection{Example 1: Brownian motion}

In this example we consider that SP $a(t, \omega) \equiv B(t, \omega)$ is the Brownian motion or standard Wiener and $t_{0}=0$. Then, it is known that $\mu_{a}(t)=0$ and $\mathbb{V}[a(t, \omega)]=1, \forall t \in \mathcal{T}=[0, T], T>0$. In addition, the covariance function is given by

$$
c_{a}(s, t)=\min (s, t), \quad(s, t) \in \mathcal{T} \times \mathcal{T},
$$

which has the following eigenvalues and normalized eigenfunctions (see [9, Chapter 2])

$$
v_{j}=\frac{4 T^{2}}{\pi^{2}(2 j-1)^{2}}, \quad \phi_{j}(t)=\sqrt{\frac{2}{T}} \sin \left(\frac{(2 j-1) \pi t}{2 T}\right), \quad j=1,2, \ldots
$$

Then, the 1-PDF of the truncated solution SP $x_{N}(t, \omega)$ is obtained substituting (35) in (9)

$$
f_{1}^{N}(x, t)=\int_{\mathbb{R}^{N}} f_{0}\left(x \prod_{j=1}^{N} \mathrm{e}^{-h_{j}(t) \xi_{j}}\right) f_{\xi_{N}}\left(\xi_{1}, \ldots, \xi_{N}\right) \prod_{j=1}^{N} \mathrm{e}^{-h_{j}(t) \xi_{j}} \mathrm{~d} \xi_{N} \cdots \mathrm{d} \xi_{1},
$$


where

$$
h_{j}(t)=\left(\frac{2 T}{(2 j-1) \pi)}\right)^{2} \sqrt{\frac{2}{T}}\left(1-\cos \left(\frac{(2 j-1) \pi t}{2 T}\right)\right) .
$$

In this example the 1-PDF of the exact solution SP, $x(t, \omega)$, can be computed taking into account that $\hat{B}(t)=\int_{0}^{t} B(s) d s \sim N\left(0 ; \sqrt{\frac{t^{3}}{3}}\right)$. Hence $\hat{B}(t) \stackrel{\text { d }}{=} \sqrt{\frac{t^{3}}{3}} Z, Z \sim \mathrm{N}(0 ; 1)$, that is, the SP $\hat{B}(t)$ has the same distribution as RV, $\sqrt{\frac{t^{3}}{3}} Z$, being $Z$ a standard Gaussian RV. Using the RVT method, it is straightforwardly to check that the 1-PDF of $x(t, \omega)$ is given by

$$
f_{1}(x, t)=\int_{-\infty}^{\infty} f_{0}\left(x \mathrm{e}^{-\sqrt{\frac{3}{3}} z}\right) f_{Z}(z) \mathrm{e}^{-\sqrt{\frac{13}{3}} z} \mathrm{~d} z,
$$

where $f_{0}\left(x_{0}\right)$ and $f_{Z}(z)$ denote the PDFs of RVs $X_{0}$ and $Z$, respectively.

In Fig. 1, we show 3D-plots of the exact 1-PDF (left) and two approximations $f_{1}^{N}(x, t)$ using (36)-(37) with $N=1$ (center) and $N=2$ (right), respectively, over the time interval $[0, T]=[0,2]$. We have taken $\xi_{j}(\omega), j=1,2$ uncorrelated standard Gaussian RVs and $x_{0}(\omega)$ a uniform RV on the interval $[0,1]$, i.e., $x_{0}(\omega) \sim \mathrm{Un}([0,1])$. As it has been assumed in the theoretical development, $x_{0}(\omega)$ is assumed to be independent of $\xi_{1}(\omega)$ and $\xi_{2}(\omega)$. Notice that this assumption has been already used in (38). In the context of this example, clearly all hypotheses H1-H4 hold (see Remarks 1 and 2 to check H3 and H4, respectively). From Fig. 1, we can see that the first and second order truncations (plots in the center and in the right, respectively) are close to the 1-PDF of the exact solution (plot in the left). This feature can be observed in detail in Fig. 2 where the exact PDF, $f_{1}(x, t)$, and the two previous approximations, $f_{1}^{1}(x, t)$ and $f_{1}^{2}(x, t)$ have been plotted in different time instants $(t=0.1,1,2)$. For sake of clarity, in Table 1 we have collected the total error, defined by the following expression (39), between the exact 1-PDF and the approximation with order of truncation $N$ at different times instants

$$
e_{N}^{\mathrm{PDF}}(t)=\int_{-\infty}^{\infty}\left|f_{1}(x, t)-f_{1}^{N}(x, t)\right| \mathrm{d} x .
$$

\begin{tabular}{|c|c|c|}
\hline$e_{N}^{\mathrm{PDF}}(t)$ & $N=1$ & $N=2$ \\
\hline$t=0.1$ & 0.010021 & 0.008682 \\
\hline$t=1$ & 0.077919 & 0.008663 \\
\hline$t=2$ & 0.005310 & 0.000832 \\
\hline
\end{tabular}

Table 1: Error measure $e_{N}^{\mathrm{PDF}}(t)$ defined by (39) for different time instants, $t \in\{0.1,1,2\}$, and truncation orders $N=1,2$, in the context of Example 1.

Finally, in Fig. 3 we compare the exact mean and the exact standard deviation with the approximations obtained by (1) using, for computing the approximations, $f_{1}^{N}(x, t)$ with $N=1,2$ instead of $f_{1}(x, t)$. From these plots we can see that approximations are good, being slower the convergence of standard deviation. The errors of these approximations are shown in Table 2. These figures have been calculated using in (1) the following expressions with $t_{0}=0$ and $T=2$

$$
e_{N}^{\mu}=\int_{t_{0}}^{T}\left|\mu_{x}(t)-\mu_{x}^{N}(t)\right| \mathrm{d} t, \quad e_{N}^{\sigma}=\int_{t_{0}}^{T}\left|\sigma_{x}(t)-\sigma_{x}^{N}(t)\right| \mathrm{d} t .
$$



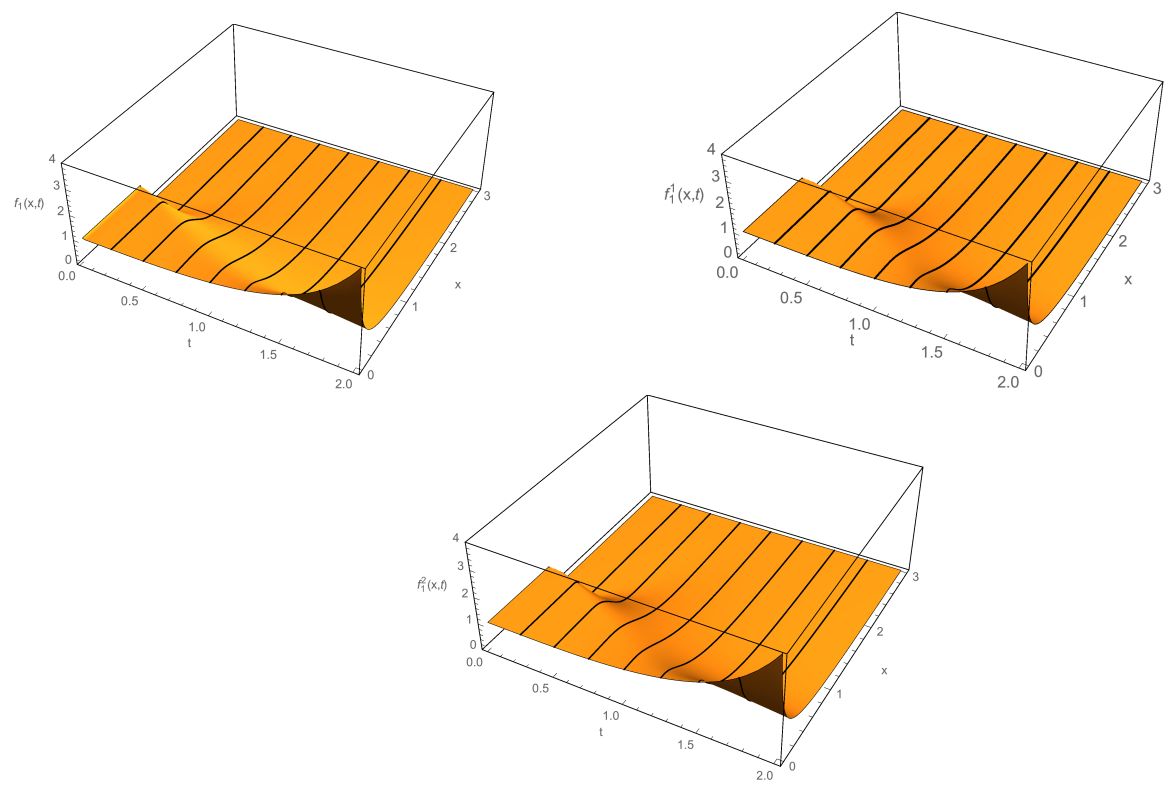

Figure 1: Left: 1-PDF of the exact solution SP given by (38). Center: 1-PDF of the first truncation given by (36)-(37) with $N=1$. Right: 1 -PDF of the second truncation given by (36)-(37) with $N=2$.
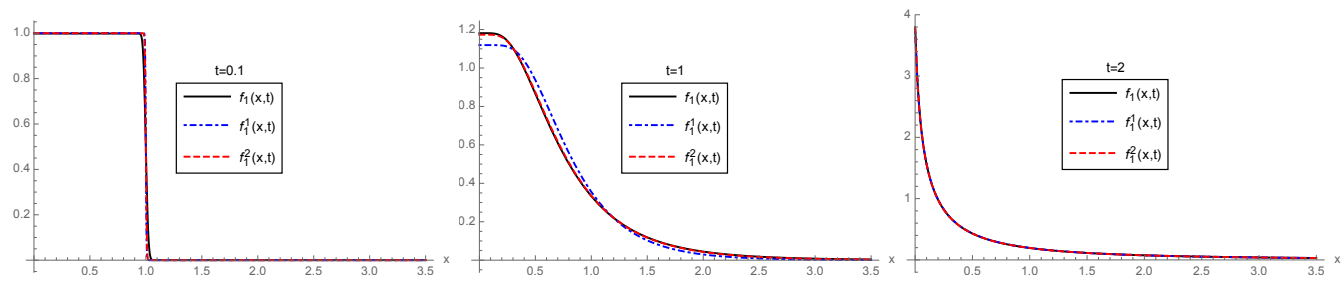

Figure 2: 1-PDF $f_{1}(x, t)$ of the exact solution SP and the two first truncations $f_{1}^{N}(x, t), N=1,2$, for different values of $t$ in the context of Example 1. Left: $t=0.1$. Center: $t=1$. Right: $t=2$.

\begin{tabular}{|c|c|c|c|c|}
\hline Error & $N=1$ & $N=2$ & $N=3$ & $N=4$ \\
\hline Mean $e_{N}^{\mu}$ & 0.055567 & 0.005541 & 0.002425 & 0.000871 \\
\hline Standard deviation $e_{N}^{\sigma}$ & 0.383975 & 0.169942 & 0.159339 & 0.151808 \\
\hline
\end{tabular}

Table 2: Values of errors $e_{N}^{\mu}$ and $e_{N}^{\sigma}$ for the mean and standard deviation, given by (40) using different orders of truncation $N$, in the context of Example 1. 

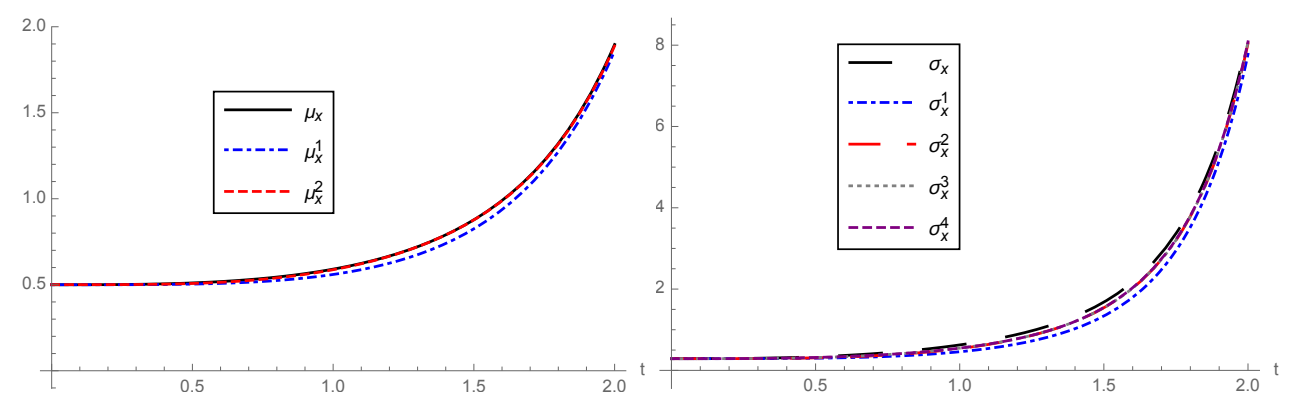

Figure 3: In the context of Example 1. Left: Exact mean $\left(\mu_{x}\right)$ of the solution and its approximations using truncations of order $N=1,2\left(\mu_{x}^{1}\right.$ and $\mu_{x}^{2}$, respectively). Right: Exact standard deviation $\left(\sigma_{x}\right)$ of the solution and its approximations using truncations of order $N=1,2,3,4\left(\sigma_{x}^{i}, i=1,2,3,4\right)$.

\subsection{Example 2: Exponential covariance}

As the KLE relies upon the covariance function $c_{a}(s, t)$ of the SP, in this example we will assume known the covariance function of SP $a(t, \omega)$ instead of giving the SP itself. Let us consider the following covariance function, usually referred to as exponential covariance,

$$
c_{a}(s, t)=\mathrm{e}^{-\frac{|s-t|}{b}}, \quad(s, t) \in \mathcal{T} \times \mathcal{T}, \quad \mathcal{T}=[-a, a], a>0,
$$

where $b>0$ is often termed the correlation length, since it reflects the rate at which the correlation decays between two times of the process. The eigenvalues and normalized eigenfunctions of the covariance function are given by [3, p.294-295]

$$
\begin{aligned}
\phi_{j}(t) & =\frac{\cos \left(z_{j} x\right)}{\sqrt{a+\frac{\sin \left(2 z_{j} a\right)}{2 z_{j}}}}, \quad v_{j}=\frac{2 c}{z_{j}^{2}+c^{2}}, \quad j \text { odd }, \\
\phi_{j}^{*}(t) & =\frac{\sin \left(z_{j}^{*} x\right)}{\sqrt{a-\frac{\sin \left(2 z_{j}^{*} a\right)}{2 z_{j}^{*}}}}, \quad v_{j}^{*}=\frac{2 c}{\left(z_{j}^{*}\right)^{2}+c^{2}}, \quad j \text { even, }
\end{aligned}
$$

being $c=1 / b$ and $z_{j}, z_{j}^{*}$ the solutions of the following transcendental equations

$$
\begin{aligned}
& c-z_{j} \tan \left(\omega_{j} a\right)=0, \quad j \text { odd, } \\
& z_{j}^{*}+c \tan \left(z_{j}^{*} a\right)=0, \quad j \text { even. }
\end{aligned}
$$

Then, considering the mean of the SP $a(t, \omega)$ is zero, the KLE of $a(t, \omega)$ is given by

$$
a(t, \omega)=\sum_{j=1}^{\infty}\left(\sqrt{v_{2 j-1}} \phi_{2 j-1}(t) \xi_{2 j-1}(\omega)+\sqrt{v_{2 j}^{*}} \phi_{2 j}^{*}(t) \xi_{2 j}^{*}(\omega)\right) .
$$


Next, we will show graphically the approximations, $f_{1}^{N}(x, t)$, of the 1-PDF, $f_{1}(x, t)$, of the solution SP, $x(t, \omega)$, to random IVP (2), being $a(t, \omega)$ represented by the KLE (43). These approximations will be constructed using expression (9) with different orders of truncation $N$.

In Fig. 4 , we have plotted $f_{1}^{N}(x, t)$ with $N=1$ and $N=2$ for two different correlation lengths, $b=0.1$ and $b=1$, over the time domain $\mathcal{T}=[-0.5,0.5]$ corresponding to $a=0.5$. Therefore, the initial time instant is $t_{0}=-0.5$. We have chosen $\xi_{j}(\omega), j=1,2$, uncorrelated standard Gaussian RVs to represent the SP $a(t, \omega)$ by KLE (43). The initial condition $x_{0}(\omega)$ is assumed to be an exponential RV with mean $1 / 4$, i.e., $x_{0}(\omega) \sim \operatorname{Exp}(4)$. Hence, by Remark 1, hypothesis H3 holds. We assume that $x_{0}(\omega), \xi_{1}(\omega)$ and $\xi_{2}(\omega)$ are independent RVs. From Fig. 4, we can observe that both $f_{1}^{1}(x, t)$ and $f_{1}^{2}(x, t)$ are very similar, then indicating quick convergence with respect to the truncation order $N$. This happens for both values of parameter $b$ over the whole space-time domain. For the sake of clarity, in Fig. 5 we show both approximations in the middle point $t=0$ of the domain $\mathcal{T}$ for $b=0.1$ and $b=1$. As an indicator of convergence, in Table 3 we have computed the following error

$$
\hat{e}_{N}^{\mathrm{PDF}}(t)=\int_{-\infty}^{\infty}\left|f_{1}^{N}(x, t)-f_{1}^{N-1}(x, t)\right| \mathrm{d} x,
$$

between consecutive approximations over the whole spacial domain at $t=0$ (middle time instant) for both values of parameter $b$.

\begin{tabular}{|c|c|c|}
\hline$\hat{e}_{N}^{\mathrm{PDF}}(0)$ & $N=2$ & $N=3$ \\
\hline$b=1$ & 0.0106515 & 0.0000164 \\
\hline$b=0.1$ & 0.0147553 & 0.0008538 \\
\hline
\end{tabular}

Table 3: Error measure $\hat{e}_{N}(t)$ defined by (44) for time instant $t=0$, and truncation orders, $N=2,3$ for $b=1$ and $b=0.1$, in the context of Example 2.

In Fig. 6 we show the mean and the standard deviation with $b=0.1$ and $b=1$ for different orders of truncation.

To account for the error, in Table 4 and Table 5 we show the following errors for $b=0.1$ and $b=1$ respectively, with $t_{0}=-0.5$ and $T=0.5$

$$
\hat{e}_{N}^{\mu}=\int_{t_{0}}^{T}\left|\mu_{x}^{N}(t)-\mu_{x}^{N-1}(t)\right| \mathrm{d} t, \quad \hat{e}_{N}^{\sigma}=\int_{t_{0}}^{T}\left|\sigma_{x}^{N}(t)-\sigma_{x}^{N-1}(t)\right| \mathrm{d} t,
$$

where $\mu_{x}^{n}(t)$ and $\sigma_{x}^{n}(t), n=N-1, N$, are approximations to the mean and the standard deviation using $f_{1}^{N-1}(x, t)$ and $f_{1}^{N}(x, t)$, respectively, instead of $f_{1}(x, t)$ in expression (1).

\begin{tabular}{|c|c|c|c|c|}
\hline error $(b=0.1)$ & $N=2$ & $N=3$ & $N=4$ & $N=5$ \\
\hline$\hat{e}_{N}^{\mu}$ & 0.0018717 & 0.0004470 & 0.0001983 & 0.0000631 \\
\hline \hline$\hat{e}_{N}^{\sigma}$ & 0.0057412 & 0.0013707 & 0.0006206 & 0.0003282 \\
\hline
\end{tabular}

Table 4: Errors $\hat{e}_{N}^{\mu}$ and $\hat{e}_{N}^{\sigma}$ for the mean and the standard deviation, defined by (45) respectively, using different orders of truncations $(N=2,3,4,5)$ and correlation length $b=0.1$, in the context of Example 2 .

From Table 3, we observe that the error $\hat{e}_{N}^{\mathrm{PDF}}(0)$ is smaller for $b=1$ than $b=0.1$. This same behaviour happens regarding the approximations of the mean and the standard deviation, namely, 

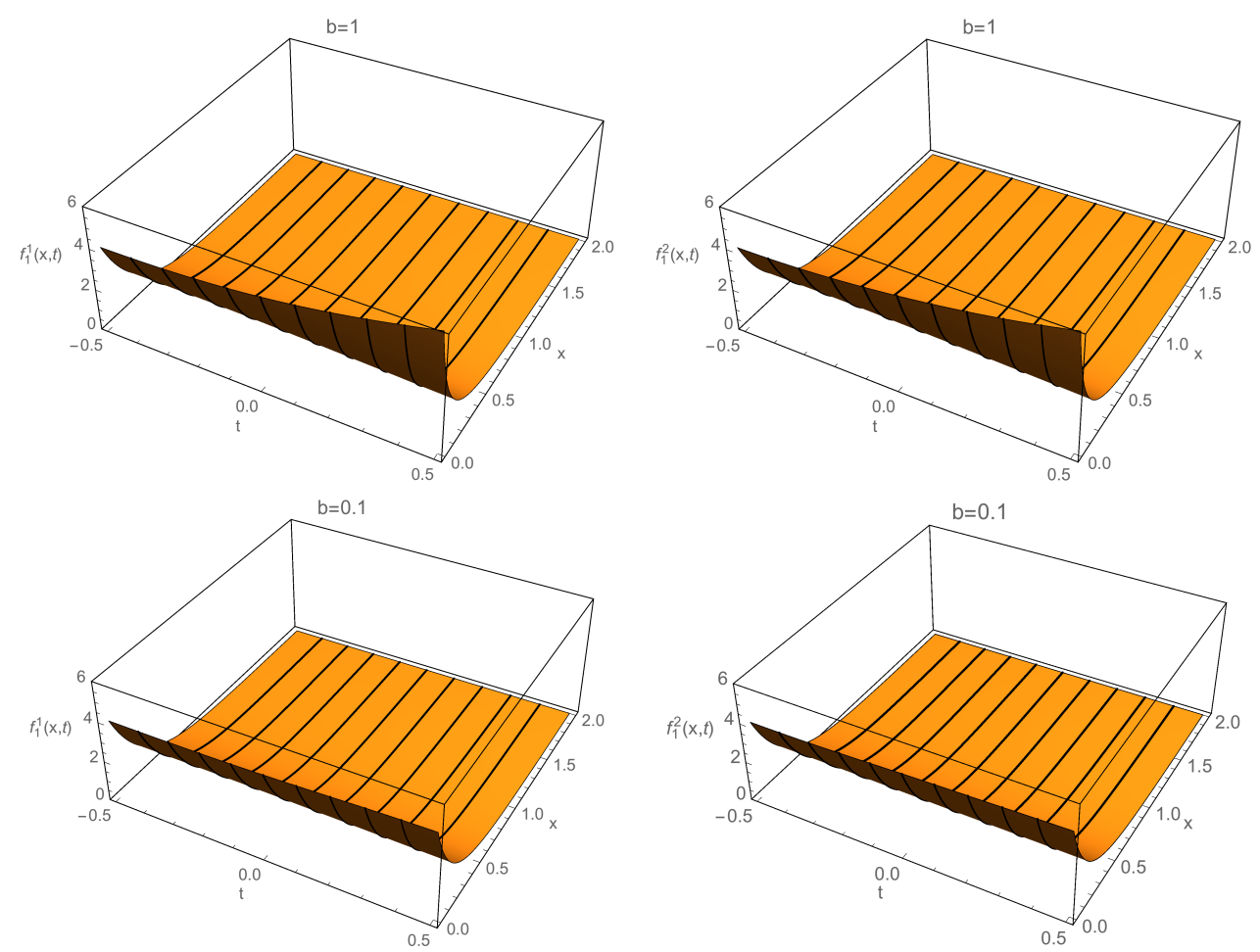

Figure 4: In the context of Example 2. Top: 1-PDF of the truncations $N=1$ and $N=2$, with $b=1$ Bottom: 1-PDF of the truncations $N=1$ and $N=2$, with $b=0.1$.
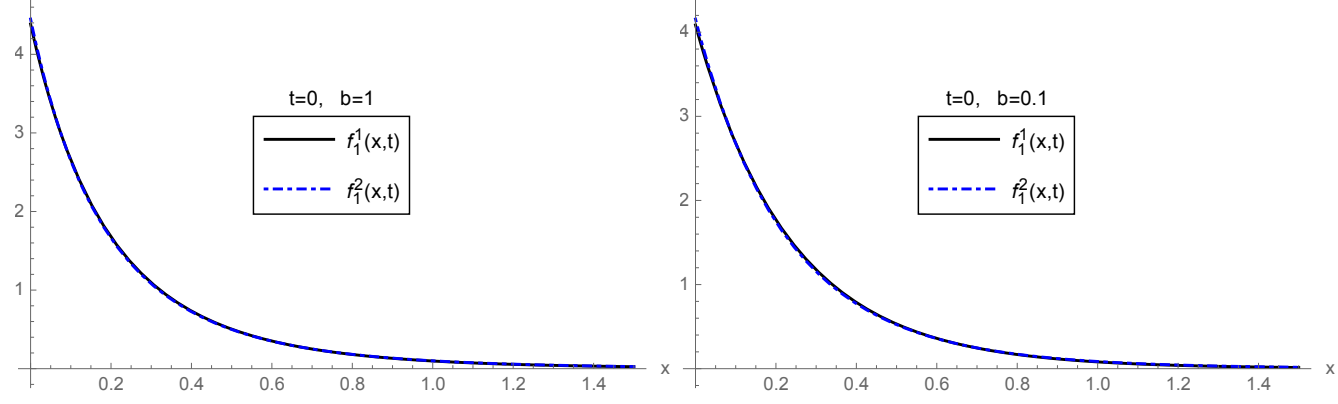

Figure 5: 1-PDF of the truncations $N=1,2$, for $t=0$, in the context of Example 2. Left: $b=1$. Right: $b=0.1$. 

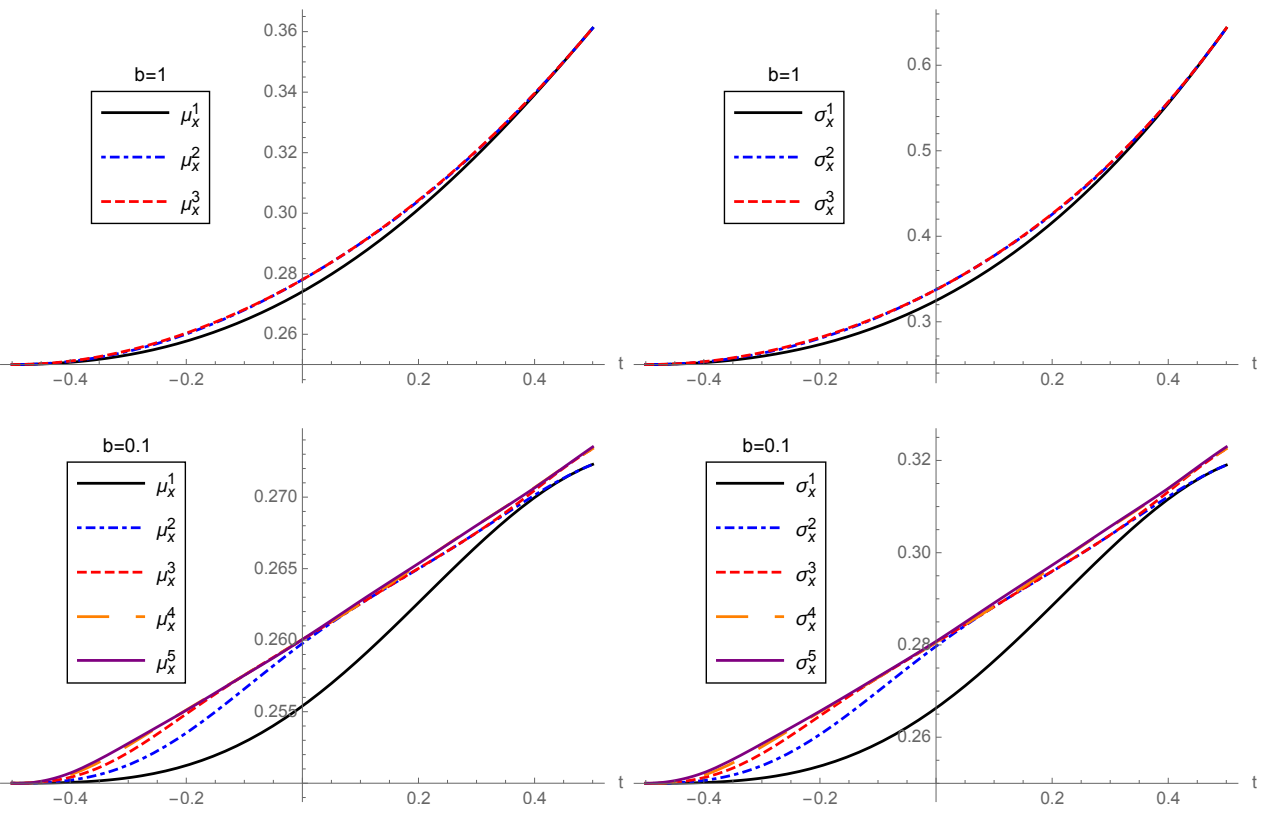

Figure 6: In the context of Example 2. Top: Approximations of the mean and standard deviation using different orders of truncation $N=1,2,3$ with correlation length parameter $b=1$. Bottom: Approximations of the mean and standard deviation using different orders of truncation $N=1,2,3,4,5$ deviation using different orders of truncation $N=1,2,3$ $b=0.1$.

\begin{tabular}{|c|c|c|}
\hline error $(b=1)$ & $N=2$ & $N=3$ \\
\hline$\hat{e}_{N}^{\mu}$ & 0.0019514 & 0.0001370 \\
\hline \hline$\hat{e}_{N}^{\sigma}$ & 0.0064182 & 0.0004473 \\
\hline
\end{tabular}

Table 5: Errors $\hat{e}_{N}^{\mu}$ and $\hat{e}_{N}^{\sigma}$ for the mean and the standard deviation, defined by (45) respectively, using different orders of truncations $(N=2,3)$ and correlation length $b=1$, in the context of Example 2. 
Table 6: First eigenvalues, $v_{j}$, of the covariance function (41) for $j=1,2, \ldots, 10$ for $b=0.1,1$ over the time interval $\mathcal{T}=[-0.5,0.5]$, in the context of Example 2.

they are better for $b=1$ than for $b=0.1$, except for $N=2$ (see Table 4 and Table 5). This result can be expected from the decay of eigenvalues $v_{j}$. In fact, it is well-known that $[3,204]$

$$
\left\|a(t, \omega)-a_{N}(t, \omega)\right\|_{\mathrm{L}^{2}\left(\Omega, \mathrm{L}^{2}(\mathcal{T})\right)}=\int_{\mathcal{T}} \mathbb{V}[a(t, \omega)] \mathrm{d} t-\sum_{j=1}^{N} v_{j} .
$$

Then, the first $N$ eigenvalues $v_{j}$ can be added to determine the truncation parameter $N$ for a given error tolerance, say $\epsilon>0$, when approximating the diffusion coefficient $a(t, \omega)$ using the KLE. Obviously, the greater the values of $v_{j}$, the smaller the value of $N$. In our context the decay of eigenvalues $v_{j}$ depends on the choice of the parameter $b$. The bigger $b$ the faster decay of $v_{j}$ and, as a consequence, a smaller value of the truncation parameter $N$ will be required to achieve the accuracy $\epsilon$ in order to approximate $a(t, \omega)$. In Table 6 , the first eigenvalues of the covariance function (41) are shown for $b=0.1$ and $b=1$ over the time interval $\mathcal{T}=[-0.5,0.5]$. These eigenvalues have been represented in Fig. 7. As it can be observed in this plot, the eigenvalues corresponding to $b=1$ decay faster than those ones corresponding to $b=0.1$. This fact is in agreement with figures collected in Table 3 (corresponding to approximation of 1-PDF) and in Tables 4 and 5 (corresponding to approximations of mean and standard deviation), where error associated to $b=1$ is smaller than to $b=0.1$, for a fixed truncation order $N$.

\begin{tabular}{|c|c|c|c|c|c|}
\hline & $v_{1}$ & $v_{2}$ & $v_{3}$ & $v_{4}$ & $v_{5}$ \\
\hline$b=0.1$ & 0.187083 & 0.156046 & 0.121154 & 0.091324 & 0.068736 \\
\hline$b=1$ & 0.738813 & 0.138000 & 0.045089 & 0.021329 & 0.012279 \\
\hline \hline & $v_{6}$ & $v_{7}$ & $v_{8}$ & $v_{9}$ & $v_{10}$ \\
\hline$b=0.1$ & 0.052403 & 0.040695 & 0.032225 & 0.025998 & 0.021333 \\
\hline$b=1$ & 0.007945 & 0.005551 & 0.004093 & 0.003142 & 0.002486 \\
\hline
\end{tabular}

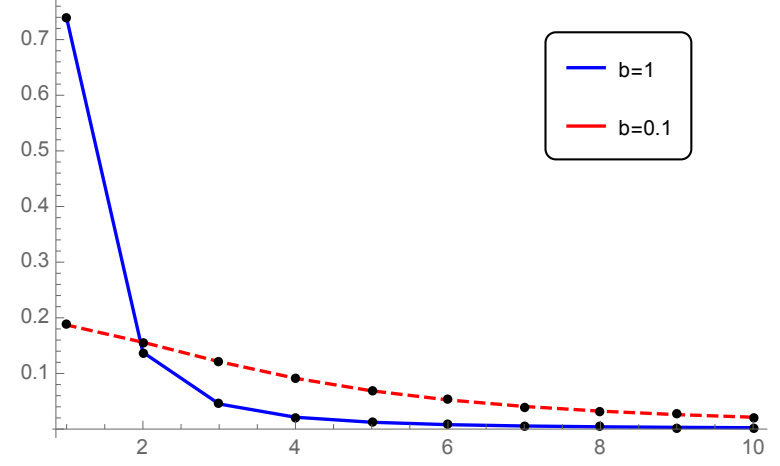

Figure 7: First eigenvalues, $v_{j}$, of the covariance function (41), in the context of Example 2. In the context of this example, where neither the exact 1-PDF nor the exact mean and standard
iation are not available, it is very interesting to establish some criterion (stopping criterion) in 
order to determine the order of truncation $N$ for a given error tolerance $\epsilon>0$. Just as an example to illustrate the way our findings can be used in this regard, if $\epsilon=10^{-3}$ then, according to Table 4 and Table 5, it is enough to take $N=3$ as the order of truncation for both values of parameter $b$, while $N=3$ and $N=4$ are required to guarantee the same accuracy for the standard deviation when $b=1$ and $b=0.1$, respectively.

\section{Conclusions}

In this paper we have constructed approximations of the first probability density function to the linear homogeneous first-order random differential equation. We have proved rigorously that these approximations are convergent under mild conditions upon the initial condition and the diffusion coefficient which are assumed to be a random variable and a stochastic process, respectively. The key idea to construct these approximations has been to combine the Random Variable Transformation technique and the Karhunen-Loève expansion. We have considered two illustrative examples showing that approximations converge rapidly. Indeed, just a few terms are need to approximate the first probability density function (1-PDF) in both examples. We have taken advantage of computing the 1-PDF to approximate both the mean and the variance in both examples. All numerical results are also satisfactory. Finally, as it has been underlined in the motivation of the paper, although the formulation of the target problem appears to be simple, the analysis does not. This is a genuine feature usually met when deterministic results are extended to the random scenario.

\section{Acknowledgements}

This work has been partially supported by the Ministerio de Economía y Competitividad grant MTM2017-89664-P. Ana Navarro Quiles acknowledges the doctorate scholarship granted by Programa de Ayudas de Investigación y Desarrollo (PAID), Universitat Politècnica de València.

\section{Conflict of Interest Statement}

The authors declare that there is no conflict of interests regarding the publication of this article.

\section{References}

[1] R. C. Smith, Uncertainty Quantification: Theory, Implementation, and Applications, Computational Science and Engineering, SIAM, Philadelphia, 2014.

[2] B. Øksendal, Stochastic Differential Equations: An Introduction with Applications, 6th Edition, Springer, New York, 2010.

[3] G. Lord, C. Powell, T. Shardlow, An Introduction to Computational Stochastic PDEs, Cambridge Texts in Applied Mathematics, Cambridge University Press, New York, 2014.

[4] P. E. Kloeden, E. Platen, Numerical Solution of Stochastic Differential Equations, 3rd Edition, Vol. 23 of Applications of Mathematics: Stochastic Modelling and Applied Probability, Springer, New York, 1999.

[5] E. Allen, Modeling with Itô Stochastic Differential Equations, Springer, New York, 2007.

[6] T. T. Soong, Random Differential Equations in Science and Engineering, Academic Press, New York, 1973.

[7] T. Neckel, F. Rupp, Random Differential Equations in Scientific Computing, Versita, London, 2013.

[8] D. Xiu, Numerical Methods for Stochastic Computations: A Spectral Method Approach, Princeton University Press, New Jersey, 2010. 
[9] R. G. Ghanem, P. D. Spanos, Stochastic Finite Elements: A Spectral Approach, Dover Publications, New York, 2003.

10] J. Strand, Random ordinary differential equations, Journal of Differential Equations 7 (3) (1970) 538 - 553. doi:http://dx.doi.org/10.1016/0022-0396(70)90100-2.

[11] T. C. Gard, Introduction to Stochastic Differential Equations, Marcel Dekker, New York, 1988.

[12] M. C. Casabán, J. C. Cortés, J. V. Romero, M. D. Roselló, Determining the first probability density function of linear random initial value problems by the Random Variable Transformation (RVT) technique: A comprehensive study, Abstract and Applied Analysis 2014-ID248512 (2014) 1-25. doi:10.1155/2013/248512.

[13] A. Hussein, M. M. Selim, Solution of the stochastic transport equation of neutral particles with anisotropic scattering using RVT technique, Applied Mathematics and Computation 213 (1) (2009) 250-261. doi:10.1016/j.amc.2009.03.016.

[14] A. Hussein, M. M. Selim, Solution of the stochastic radiative transfer equation with Rayleigh scattering using RVT technique, Applied Mathematics and Computation 218 (13) (2012) 7193-7203. doi:10.1016/j.amc.2011.12.088.

[15] L. T. Santos, F. A. Dorini, M. C. C. Cunha, The probability density function to the random linear transport equation, Applied Mathematics and Computation 216 (5) (2010) 1524-1530. doi:10.16/j.amc.2010.03.001.

[16] F. A. Dorini, M. S. Cecconello, L. B. Dorini, On the logistic equation subject to uncertainties in the environmental carrying capacity and initial population density, Communications in Nonlinear Science and Numerical Simulation 33 (2016) 160-173. doi:10.1016/j.cnsns.2014.12.016.

[17] M. C. Casabán, J. C. Cortés, A. Navarro-Quiles, J. V. Romero, M. D. Roselló, R. J. Villanueva, A comprehensive probabilistic solution of random SIS-type epidemiological models using the Random Variable Transformation technique, Communications in Nonlinear Science and Numerical Simulation 32 (2016) 199-210. doi:10.1016/j.cnsns.2014.12.016. 